\title{
Linear Control of Highly Flexible Aircraft based on Loop Separation
}

\author{
Pedro J. González R. ${ }^{1}$, Flávio J. Silvestre ${ }^{2}$, Pedro Paglione ${ }^{3}$ \\ Instituto Tecnológico de Aeronáutica, São José dos Campos, SP, 12228-900, Brazil \\ Alexander Köthe ${ }^{4}$ \\ Technische Universität Berlin, Marchstraße 12, 10587 Berlin, Germany \\ Zi Yang Pang, ${ }^{5}$ and Carlos E. S. Cesnik ${ }^{6}$ \\ University of Michigan, Ann Arbor, MI, 48109-2410
}

\begin{abstract}
The outcome of highly flexible aircraft requires new approaches in control design. In this research, we apply the loop separation concept, which consists in two control loops. The inner loop is capable of stabilizing the plant of the flexible aircraft, while is holding shape of the trimmed structure. Once the highly flexible aircraft is artificially transformed in a slightly flexible aircraft, the second loop or outer-loop is designed according to conventional, rigid-body-based control. Three control approaches were evaluated in the inner loop: LQG/LTR, LQR with output feedback and a direct integration approach. The direct integration approach with uncoupled gains presented better performance. The outer loops for speed, heading, sideslip angle and altitude were estimated using non-smooth optimization techniques and they are capable of attaining the commanded reference with low control energy and inside the maneuver requirements, while the inner loop is capable of reducing the elastic strains of the wing.
\end{abstract}

\section{Nomenclature}

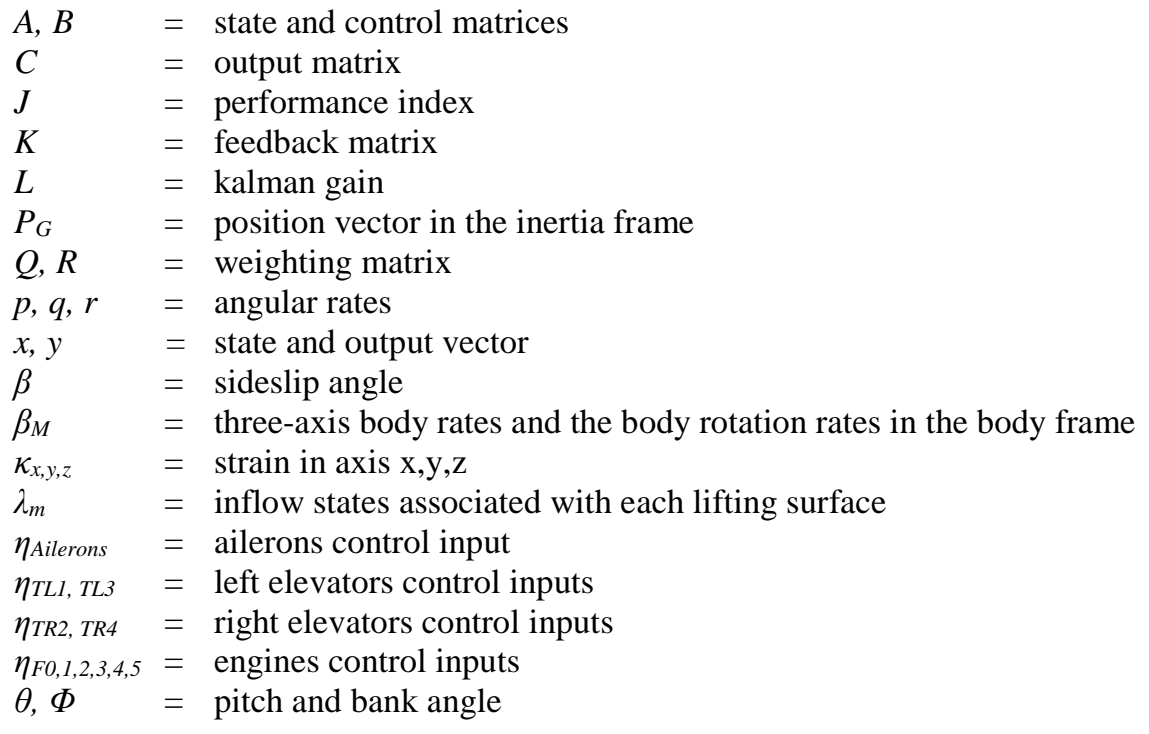

${ }^{1}$ PhD Candidate, Aerospace Engineering Division, São José dos Campos, Member AIAA

${ }^{2}$ Assistant Professor, Aerospace Engineering Division, São José dos Campos, Member AIAA.

${ }^{3}$ Professor, Aerospace Engineering Division, São José dos Campos, Member AIAA.

${ }^{4}$ Research Assistant and Ph.D. candidate, Department of Flight Mechanics, Flight Control and Aeroelasticity.

${ }^{5} \mathrm{PhD}$ Candidate, Department of Aerospace Engineering, MI, Member AIAA.

${ }^{6}$ Professor, Department of Aerospace Engineering, MI, Fellow AIAA.

American Institute of Aeronautics and Astronautics 


\section{Introduction}

$\mathrm{T}$ HE high altitude, long endurance vehicles are in consideration for missions that include long-term flights, in order to replace low altitude satellites. Aircraft like DARPA Vulture ${ }^{1,}{ }^{\text {SHAMPO }}{ }^{2}$ and $\mathrm{Zephyr}^{3}$ are examples of systems designed with this purpose. The principal technical aspects that must to be evaluated to improve these aircrafts performance are propulsion, aerodynamic, structural and control efficiency ${ }^{4}$. The wing is responsible for a high percentage of the aircraft drag. To gain aerodynamic efficiency the induced drag is reduced using high aspect ratio wings. The maximum flight endurance is obtained by flying as close as possible to the minimum power condition, causing a reduction in the fuel or energy consumption. To increase structural efficiency it is required to keep the structure as light as possible, in addition it has to be strong enough to handle maneuvers and gust loads. The control efficiency seeks a long endurance autonomous operation with a minimum or non-human intervention. The installed aircraft control law has the objective to accomplish a complete mission under various weather conditions. Additionally, the control law must be feasible to be implemented on board the aircraft, and it shall have a flexibility to be online adaptive, according to mission modifications during the execution.

The design of flight control laws is regularly based on the rigid-body approximation of the aircraft flight dynamics. However, increasing aspect ratio and structural efficiency leads to more flexible structures, which is accompanied by a reduction in the frequencies of aeroelastic modes. This leads to a coupling of aeroelastic and flight dynamics ${ }^{5}$. Control laws developed only using rigid body dynamics will have degraded performance due to modeled dynamics. Meanwhile, the lack of deformation control considering may lead to structural damage, like in the Helios incident ${ }^{6}$.

The objective of this paper is to present a methodology for flight control law design of flexible aircraft. It is based on a two-loop concept: an internal loop to augment stability while keeping aircraft shape with small elastic displacements; and an external loop to track altitude, velocity and heading. The concept is applied to X-HALE, a flight test platform representative of a very flexible aircraft developed by the Active Aeroelasticity and Structures Research Laboratory of the University of Michigan to study the complex flexible aircraft dynamics.

\section{X-HALE state of the art}

X-HALE was initially designed to have a span of $8 \mathrm{~m}$ but flight tests indicated that the high flexibility characteristics showed up already in the configuration with $6 \mathrm{~m}$ wingspan. Currently, X-HALE (see Fig. 1) operates in two configurations with wingspan of $4 \mathrm{~m}$ and $6 \mathrm{~m}$, both with a wing chord of $0.2 \mathrm{~m}$, constant span-wise. It has five pods ( 3 pods in the 4-m-configuration) equally distributed along the span containing the engines and payload.

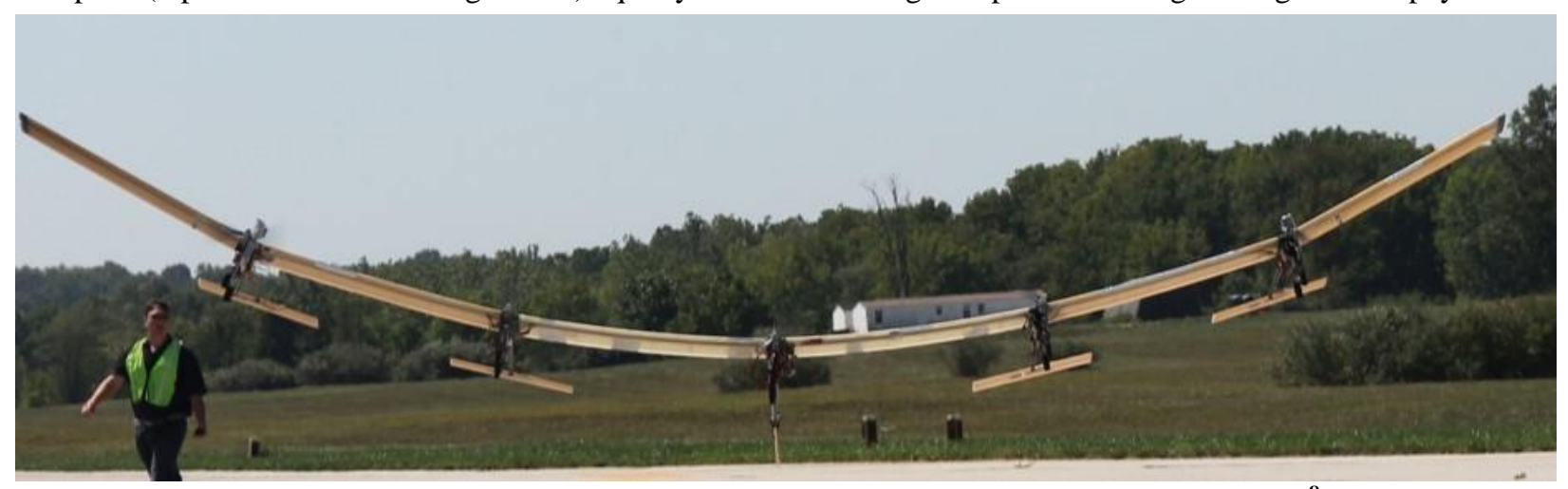

Figure 1. Experimental High Altitude Long Endurance Aircraft (X-HALE) ${ }^{8}$.

Two groups of sensors are installed on board, one to monitor the aeroelastic behavior, composed by: a group of cameras, strain gauges and accelerometers; and a second group consisting of sensors to monitor, control inputs and flight condition.

Due to the risk involved in the early flight of X-HALE, a lightly instrumented airplane was build. X-HALE Risk Reduction Vehicle (RRV) was designed to verify the aeroelastic properties of the aircraft and to understand its flight behavior before a fully instrumented X-HALE could be tested ${ }^{8}$. X-HALE Aeroelastic Test Vehicle (ATV) is the fully instrumented aircraft, equipped with a camera system to measure the wing deformation in flight ${ }^{9}$. Two pairs of cameras were installed on the center pod facing the wing tips, and three pairs of LED markers were placed on top of each section of the wing. The cameras are able monitor the variation of position of the LED markers; the deformed wing shape can be obtained by post-processing of recorded images. In the new version of ATV, each pod is additionally equipped with inertia measurement units. 
In 2015, the Laboratory of New Concepts in Aeronautics (LNCA) of the Aeronautics Institute of Technology (ITA) started a research project funded by the Brazilian funding agency FINEP and EMBRAER, in cooperation with UMich, with two major objectives: validation of coupled models of flight and aeroelastic dynamics with different fidelity levels; and control law design for flexible aircraft. Within this research project, X-HALE is currently being built in Brazil.

\section{A. Description of the linearized model of X-HALE dynamics}

The 6-m span configuration with basic instrumentation (RRV) has been used in this work. The model represents the six panels with their respective booms, stabilizers and pods, it possesses three ventral fins on the pods to increase lateral stability. The outer sections of the wing have a dihedral angle of installation of 10 degrees.

The model was built in the University of Michigan Nonlinear Aeroelastic Simulation and Toolbox (UM/NAST). UM/NAST uses a strain based formulation of elastic equations of motion, joint with rigid body equations and is capable of simulating rigid body, linearized and nonlinear aircraft dynamics. UM/NAST provides a suitable plant representation for control design ${ }^{7}$. For the structural model, four inboard 1-meter sections are modelled using two flexible elements, while outboard dihedrals are modelled using four flexible elements. Tails are modelled using two rigid elements; booms, pods and tails are rigid. The wings, fins and tails, are represented in the aerodynamic model as lifting surfaces, while the booms are modelled as non-lifting surfaces.

The current model of the airplane has 344 states, the linearized model is of the form:

$$
\begin{aligned}
& \dot{x}=A x+B u \\
& y=C x+D u
\end{aligned}
$$

Where $A$ is the state matrix, $B$ is the input matrix, $C$ is the output matrix, $x$ is the state vector, $y$ is the measured control output, $u$ is the control input. The state vector is defined as

$$
x=\left[\begin{array}{llllll}
\varepsilon_{n} & \dot{\varepsilon}_{n} & \beta_{M} & \zeta & P_{G} & \lambda_{m}
\end{array}\right]^{T}
$$

$\varepsilon_{n}$ is a $4 \mathrm{n}$ by 1 vector, where $n$ is the number of flexible elements, containing $x$-deformation $\varepsilon_{n}$ and strain in all axes, $\kappa_{\mathrm{x}}, \kappa_{\mathrm{y}}$ and $\kappa_{\mathrm{z}}$; for the $i$-th flexible element. For the body frame $B, x$ is the direction of the wing span, $y$ is chord wise and $z$ complete the axes been positive upwards (see Fig. 2).

$$
\varepsilon_{n i}=\left[\begin{array}{llll}
\varepsilon_{x} & \kappa_{x} & \kappa_{y} & \kappa_{z}
\end{array}\right]^{T}
$$

The $\beta_{M}$ vector consists in the three-axis body rates and the body rotation rates in the body frame. $\zeta$ is the Euler angle vector. The position vector in Earth reference frame is $P_{G}$ and $\lambda_{\mathrm{m}}$ is the six inflow states associated with each lifting surface. It is a $6 m$ by 1 vector where $m$ is the number of lifting surfaces defined.

The aircraft has ten control inputs: the four horizontal stabilizers $\left(\eta_{T L 1}, \eta_{T L 2}, \eta_{T R I}\right.$ and $\left.\eta_{T R 2}\right)$, the ailerons $\left(\eta_{A I L}\right)$ and the five engines $\left(\eta_{F 0}, \eta_{F 1}, \eta_{F 2}, \eta_{F 3}, \eta_{F 4}\right)$ as is presented in Eq. (4). Figure 2 illustrates the linearized model of X-HALE with wings and control inputs.

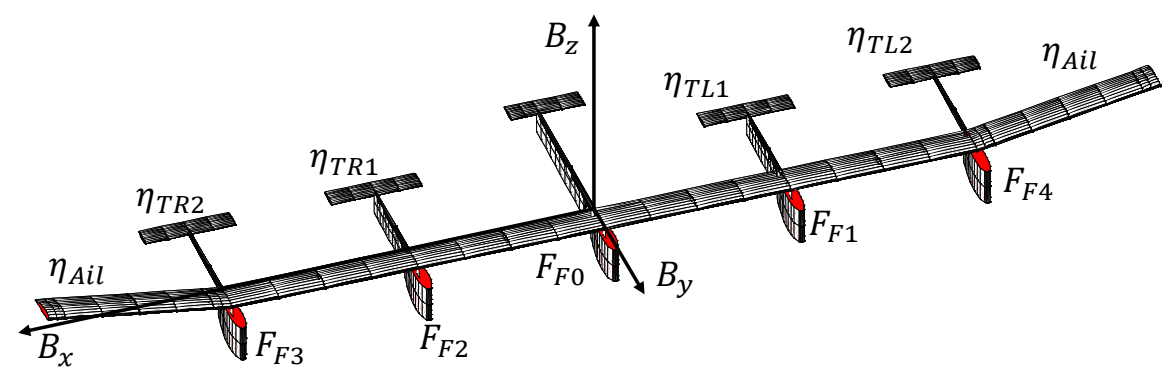

Figure 2. Identification of the linearized model of the X-HALE. 


$$
u=\left[\begin{array}{llllllllll}
\eta_{T L 1} & \eta_{T R 1} & \eta_{T L 2} & \eta_{T R 2} & \eta_{A i l} & \eta_{F 0} & \eta_{F 1} & \eta_{F 2} & \eta_{F 3} & \eta_{F 4}
\end{array}\right]^{T}
$$

The linearization of the X-HALE was performed over its trim condition at a flight speed of $14 \mathrm{~m} / \mathrm{s}$ and $30 \mathrm{~m}$ of altitude. For this condition, its angle of attack is 1.251 degrees, the thrust per engine $1.336 \mathrm{~N}$ and the elevators deflection 5.161 degrees.

\section{Requirements of the Flight Control Law}

\section{A. Flight Envelope}

The flight velocity margins for the X-HALE range from $12 \mathrm{~m} / \mathrm{s}$ to $20 \mathrm{~m} / \mathrm{s}$. The flight nominal speed is $14 \mathrm{~m} / \mathrm{s}$ at $30 \mathrm{~m}$ altitude. The range of gust speed applied during the simulations is from $4 \mathrm{~m} / \mathrm{s}$ to $5 \mathrm{~m} / \mathrm{s}$, in periods of $2 \mathrm{~s}, 4 \mathrm{~s}$ an 8 s of duration, as used by Dillsaver (2013) ${ }^{10}$.

\section{B. Flying Qualities Requirements and Maneuver Capabilities}

No certification guidelines have been developed for HALE aircraft, in terms of operational requirements and performance specifications. We use here, when applicable, the standard MIL-STD-1797A norm as a reference therefore ${ }^{11}$.

There are no official standards for operational requirements nor control performance specifications for this type of aircraft. Based in Shearer and Cesnik $(2008)^{12}$, the X-HALE is treated as a large land-based transport type aircraft, class III-L.

The maximum bank angle required must be determined by the bank angle necessary to make a standard 2 minute turn with 3 degrees per second rate. Due to the scale of the X-HALE this value should be tuned after seen the autopilots capabilities and the aircraft dynamics response.

Longitudinal requirements are not established in the standards but Shearer and Cesnik (2008) stated that a maximum climb rate of $10.16 \mathrm{~m} / \mathrm{s}$ at sea level with maximum gross weight should be sufficient for this kind of aircraft $^{12}$. This value must also be adapted for X-HALE scale.

For the initial evaluation of the controllers three types of maneuvers are normally proposed..$^{12,13}$

- altitude chance with wings levelled

- steady levelled turn starting from a zero bank angle

- climbing turn

All maneuvers shall be executed out of any flutter, LCO or divergence speed ${ }^{12}$, with a maximum control deflection \pm 30 degrees.

\section{Assumptions for control architecture}

In previous studies, Dillsaver et al. (2013) used an architecture consisting of a stable inner loop with the linear and angular velocities, augmented with appropriate error states. The outer loop consists of a nonlinear transformation of the commanded flight path angle and its rate into commanded linear and angular velocities, as well as an outer loop controller. The inner loop controller was tuned first, and once adequate performance was obtained tracking $\beta_{M}$ values, the outer loop was tuned ${ }^{13}$.

Two different control structures were implemented in the outer loop of Dillsaver et al. (2013): a PID and a sliding mode controller. The inner loop controllers were designed using dynamic inversion, Linear Quadratic Regulator (LQR), and a Linear Quadratic Gaussian (LQG) control on a reduced order linear model of the aircraft ${ }^{13}$.

In this work, the two-loop concept is also used, the inner loop is in charge of stabilizing the plant of the flexible aircraft by ensuring all poles be located in the left side of the imaginary axis and searching a small amount of control consumptions as well a relatively high damped response in the time domain. The inner loop is also in charge of keeping the shape of the trimmed structure. It is important to remember that the initial condition of the simulation is the trimmed deformation of the airplane. The control system is designed with the objective of keeping this shape and trying to suppress the wing deformation relative to the initial deformation condition. Unlike Dillsaver (2013), no dynamic inversion will be used in this controller, the whole gain matrix of the inner loop is estimated in the same iteration. Two approaches were used: a first one that couple longitudinal, lateral and wing shaping stabilization and a second one, which separate or uncouple longitudinal motion control from lateral-directional and wing shape control.

Once the inner loop is stable and capable of holding the wing shape, the outer-loop is designed with a conventional compensator approach. These compensators were used to generate the command inputs for height, velocity, sideslip and heading angle tracking. Since the flight control law shall be programmed in the aircraft board computer, an important requirement of the resulting flight control system is the simplicity of the architecture and its 
feasibility to be integrated in real time. Figure 3 shows schematically the controller architecture explored in the paper. The inner and outer loops will be explained in detail in the next sections.

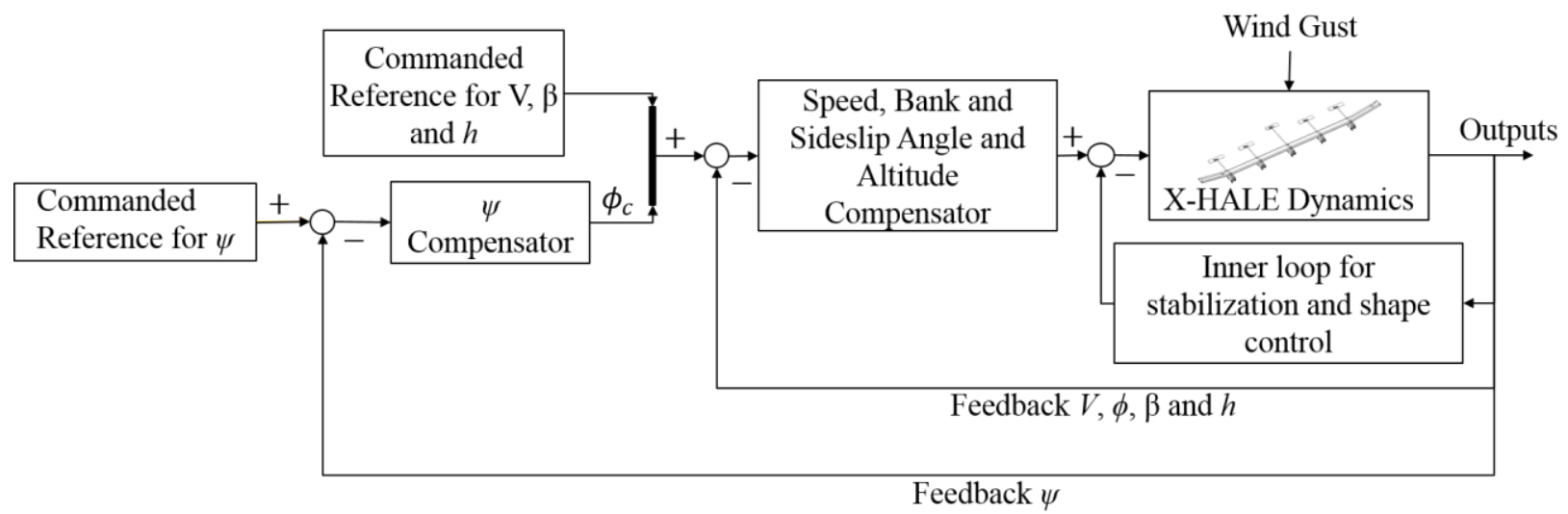

Figure 3. Structure for the control system of the X-HALE.

\section{Inner loop controller}

One of the most important considerations for the design of the autopilots is viability of its implementation. The measured outputs are pitch angle $\theta$, angular rates $p, q$ and $r$ for the aircraft flight dynamic control. For structural control or shape holding, bending and torsion strains are available on the center of each wing panel.

The controller signals of the X-HALE inner loop are mixed in order to attain the flight requirements. For longitudinal control, the central elevators are activated together. For lateral directional control and wing shape, the outer elevators and the ailerons are used, as shown in Eq. (5).

$$
u=\left[\begin{array}{llll}
\eta_{T R 2}+\eta_{T L 1} & \eta_{T R 4} & \eta_{T L 2} & \eta_{A I L}
\end{array}\right]^{T}
$$

The inner loop feedback signals are proportional to the measured feedback variables. The proportional gains are scheduled according to three techniques to know: Linear Quadratic Gaussian with Loop Transfer Recovery, Linear Quadratic Regulator with output feedback and a direct integration approach.

\section{A. Linear Quadratic Gaussian with Loop Transfer Recovery (LQG/LTR)}

The LQG/LTR technique allows the inner loop to regulate performance and control energy. It is capable to take into account process disturbances and measurement noise. This is a control approach for the design of robust aircraft control systems. It depends on full state-feedback design, followed by the design of an observer that allows complete recovery of the guaranteed robustness properties of the linear quadratic regulator with state feedback ${ }^{14}$. The idea of applying this control structure to make an inner loop for the X-HALE was based on the feasibility of estimated states of the aircraft with the kalman filter based on the measurable outputs.

The structure of the controller is presented in Fig. 4, where $w$ is the measurement noise and $v$ is the disturbance input, the Kalman gain is $K$ and the feedback matrix of the system in state space form is $L^{15}$. A state observer is obtained by producing a state estimate $\hat{x}$.

It is possible to combine the controller and the state observer by putting

$$
u=-L \hat{x}+u^{*}
$$

Where $u^{*}$ is called external input. To analyze the effect of any pair of $L$ and $K$ in the observer dynamics stability, we can write 


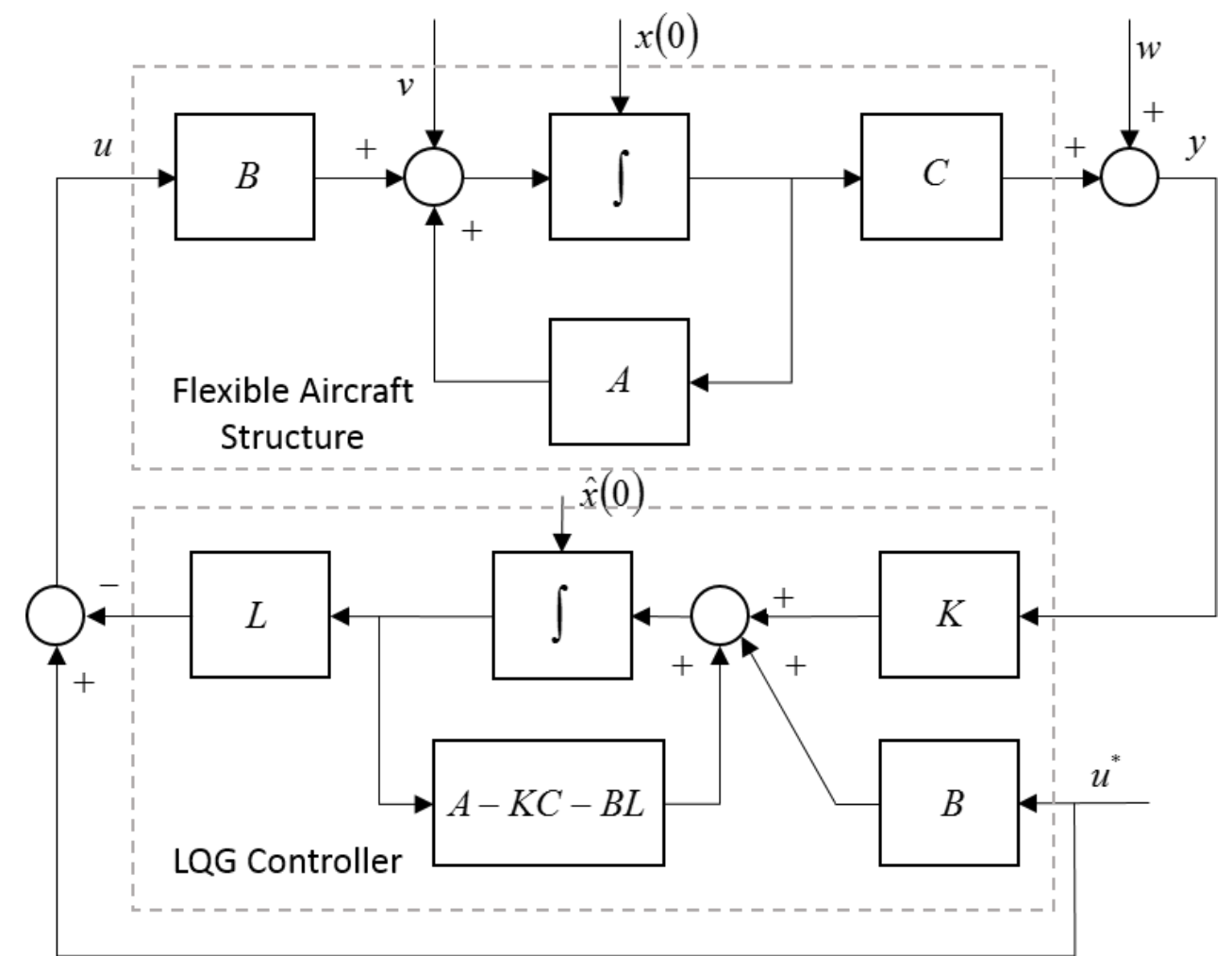

Figure 4. Inner-loop system based in LQG robust controller

$$
\begin{aligned}
& |s I-A+B L|=0 \\
& |s I-A+K C|=0
\end{aligned}
$$

Using Eq. (7) and Eq. (8) marginally stable points are obtained. The system is expressed with the next set of equations

$$
\begin{aligned}
\dot{x} & =A x+B u+v \\
y & =C x+w \\
u & =-L \hat{x}+u^{*} \\
\dot{\hat{x}} & =A \hat{x}+B u+K(y-C \hat{x})
\end{aligned}
$$

Feedback controller is given by

$$
\hat{C}(s)=L(s I-A+K C+B L)^{-1} K
$$

where $L:=S C^{\prime} Q$ and $S$ is a solution to a dual algebraic Riccati equation

$$
A S+S A^{\prime}+B R^{-1} B^{\prime}-S C^{\prime} Q C S
$$

$Q$ and $R$ matrices were tuned with the Bryson's method of diagonal weighting, based on the square of the maximum expected values of the state and control variables ${ }^{14}$. The gain matrix obtained in this problem is a completely coupled gain. 
Poles of the controller are given by $A-K C-B L$. The control system was built using the internal routines of Matlab based on the separation principle, the lqr routine was used for the estimation of the controller and the routine kalman for the estimation of the observation matrix.

\section{B. Linear Quadratic Regulator (LQR) with output feedback}

The LQR problem consists in finding a feedback coefficient matrix $K$, that given a linear system such as Eq. (1) and a control signal $u=-K y$, minimizes the magnitude of the performance index $J^{14}$. Introducing the control signal expression in Eq. (1), the close loop system is obtained

$$
\dot{x}=(A-B K C) x \equiv A_{c} x
$$

where $A_{c}$ is the close loop system. The performance index is expressed as

$$
J=\frac{1}{2} \int_{0}^{\infty}\left[x^{T} Q x+u^{T} R u\right] d t
$$

The minimization of the quadratic performance index of Eq. (13) leads to the solution of two associated Lyapunov equations, given by Eq. (14):

$$
\begin{aligned}
& 0=A_{C}{ }^{T} P+P A_{c}+Q-P B R^{-1} B^{T} P \\
& 0=A_{c} S+S A_{C}^{T}+X
\end{aligned}
$$

The gain matrix $K$ is then obtained based on matrices $P$ and $S$ as:

$$
K=R^{-1} B^{T} P S C^{T}\left(C S C^{T}\right)^{-1}
$$

To obtain the gain for minimizing the performance index is necessary to solve the three equations coupled. This make the solution only iteratively possible.

Assuming that the close loop system is stable the performance index turns into

$$
J=\frac{1}{2} x^{T}(0) P x(0)=\frac{1}{2} \operatorname{tr}(P X)
$$

where $X=x(0) x(0)^{T}$. The structure of the LQR controller with output feedback is presented in Fig. 5 . The gain obtained in this process could be coupled or uncoupled. A gain matrix is coupled when the entire controller matrix has influence in all outputs. An uncoupled control matrix means that different control actions are related to different groups of variables. The central elevators control pitch rate and pitch angle; meanwhile yaw rate, roll rate and elastic strains are hold by the outer elevators and the ailerons. Resulting gain matrix is a 4 x 16 matrix.

\section{Direct Integration Approach}

A direct integration approach (DIA) technique that minimizes the spectral abscissa of the closed loop state matrix was considered and applied in this work. The stabilization problem could be written as an unconstrained optimization problem

$$
\min (A-B K C)
$$

The closed loop stability is satisfied when the optimization result (Eq. 17) is less than 0 . To avoid convergence to a local minimum a performance index $f$ is fixed. This performance index is based in a relation between the natural frequencies $\left(\omega_{n}\right)$ and the damping ratios $(\zeta)$ of the system.

The performance index is of the form

American Institute of Aeronautics and Astronautics 


$$
f=\frac{r m x}{\exp (z m n)}
$$

where

$$
\begin{aligned}
& r m x=\max \left(-\zeta \omega_{n}\right) \\
& z m n=\min (\zeta)
\end{aligned}
$$

The structure used for this controller is the same presented in Fig. 5. As in LQR controller case, the non-smooth gain was obtained in both coupled and uncoupled forms. This means that the central elevator with only be acting to stabilize $p$ and $\theta$, while $q$ and $r$ will be controller by the outer elevators and ailerons.

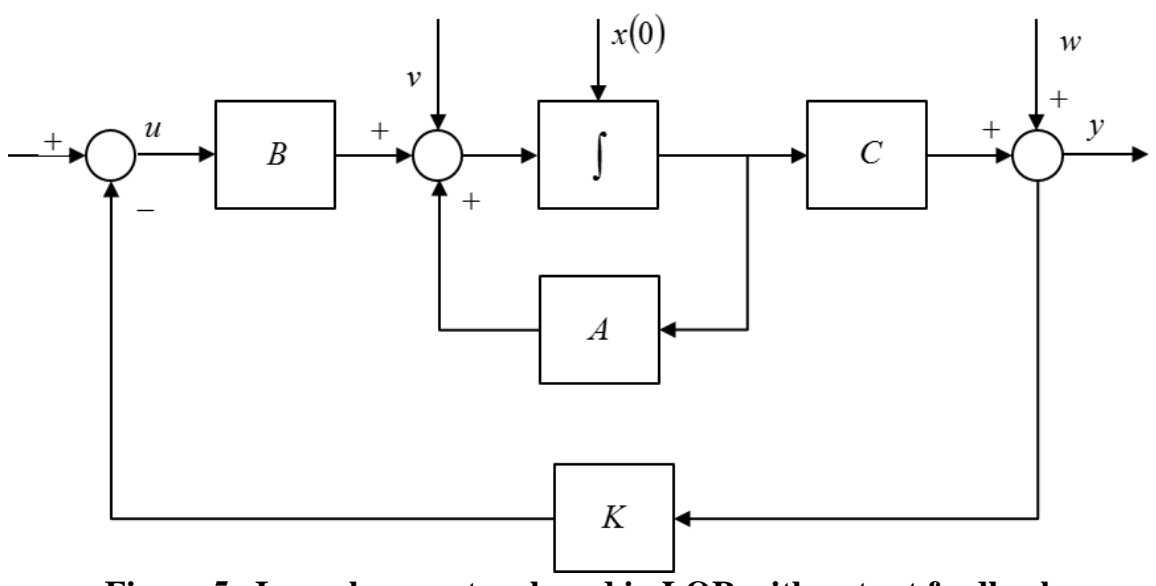

Figure 5. Inner-loop system based in LQR with output feedback.

\section{Inner loop Results}

The results presented in this section correspond to the linear simulation of X-HALE after a perturbation that increases its pitch angle in 5 degrees. The simulation was performed in Simulink and the integration of the system was done with the solver for stiff differential equations and differential algebraic equations, variable order method routine in Matlab. Figure 6 illustrates the variation of the pitching and bank angle in time domain. All control systems are able to stabilize the system. It is seen that the LQG control system has a lower damping than the LQR and the non-smooth control. It is able to attain the stationary state but after a very oscillatory transient.

The LQR and DIA controls are able to stabilize the aircraft much quicker. It is evident that the controllers with uncoupled gains get to the stationary state sooner and in a smoother way. The same tendency is observed for the bank angle disturbance. With uncoupled gains, the controllers develop smaller variation around the equilibrium condition than with coupled gains. The LQR uncoupled controller presents higher oscillatory motion, while the DIA shows a low frequency, highly damped oscillation. Is possible to notice the impact of uncoupling the gains; the control systems present a better time response. The LQG controller was synthesized with a full state observer and coupled gains. It is stable, but the performance is not sufficient to hold heading after the perturbation.

Figure 7 presents the angular rate components of X-HALE during the same maneuver. Once again, it is interesting to point out that the DIA uncoupled controller has a highly damped response, reaching stationary state with less overshoot and quicker than the other control strategies. 

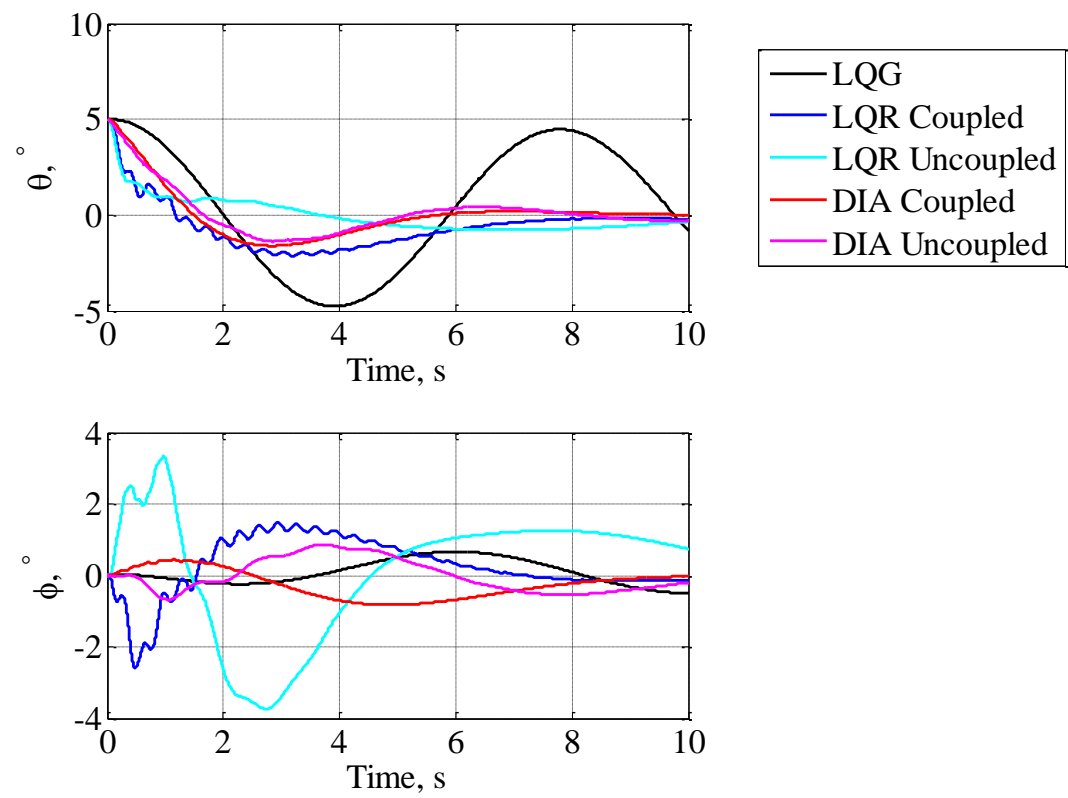

Figure 6. Pitch and bank angle in time domain.
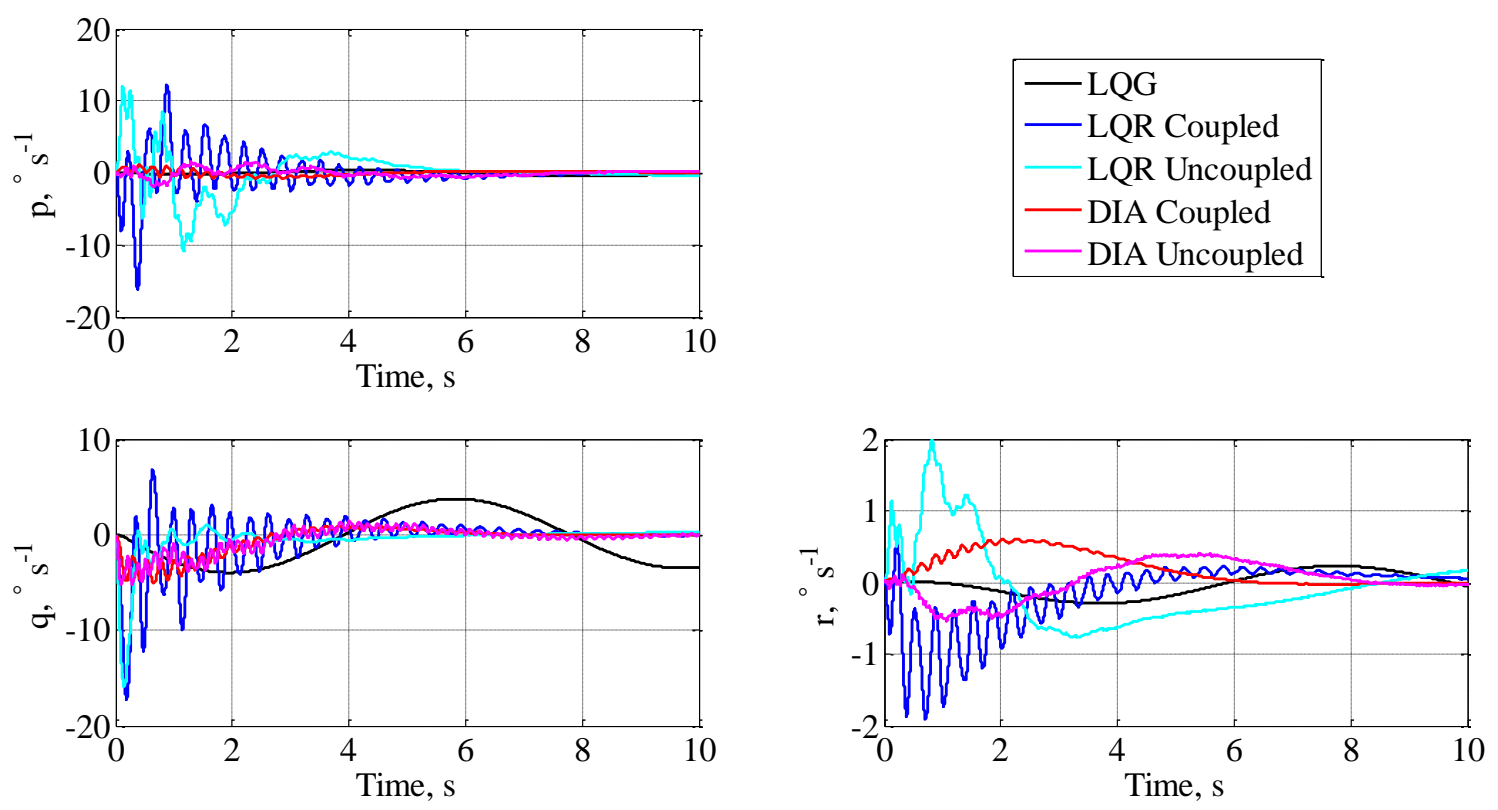

Figure 7. Aircraft velocities in time domain.

Figure 8 shows the control surface requirements to compensate the variation of the initial condition. Both inner elevators are deflecting together. The LQG has a smaller control surface deflections than the other controllers. The LQR controller requires higher control to compensate than the DIA. The DIA coupled controller needs less control surface deflection to compensate than the uncoupled. The external elevators and the ailerons move independently to return to the undisturbed flight condition, while keeping elastic strains as low as possible.

One of the objectives of the inner loop is to act over the strains, keeping the airframe as close as possible to the undeformed condition. Figures 9, 10 and 11 illustrate the strain response on the six panels of the X-HALE. All the 

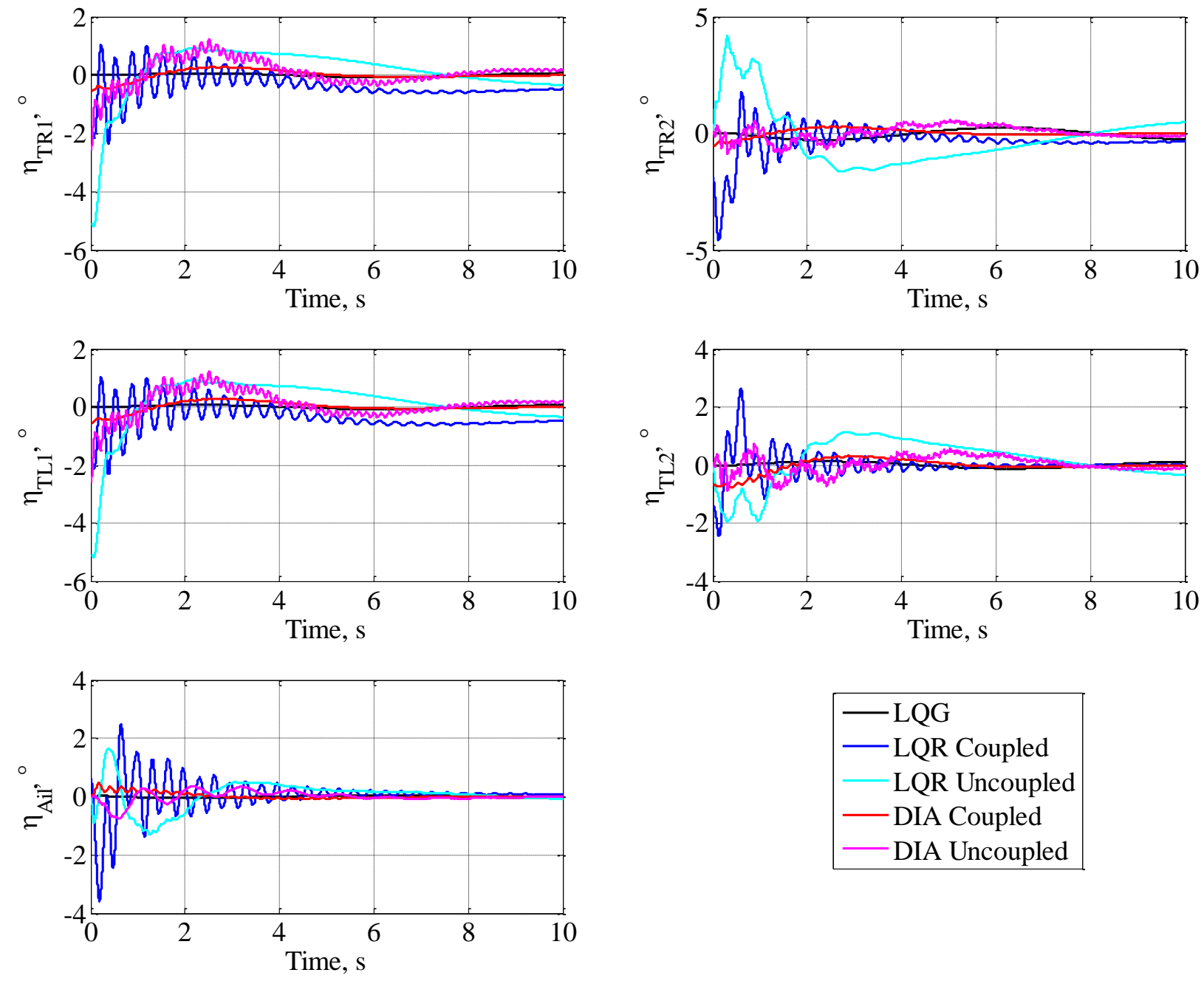

Figure 8. Control surface deflections in time domain.

controllers are capable to keep the strains in a low level. The LQG controller has a better performance in reducing strain amplitudes compared to other controllers. Following the LQG, the DIA uncoupled controller is more efficient than the other three controllers. It seems to offer a good trade-off in terms of global response and overall strain reduction. The strain in Fig 9 to 11 were measured on both wings (left and right). The sub-indexes on the strains in those figures represents in which wing panel and element they were measured.

Finally, Fig. 12 shows the variation of height. The LQG is slightly damped and oscillates around the equilibrium point. The LQR uncoupled and coupled inner loops have a damped response and a low settling time.

In general, the uncoupled controllers are capable of returning X-HALE to the equilibrium point faster and with less overshoot and higher damping. Similarly, decoupling the gains reduces the control requirements to hold equilibrium condition and to keep strains in the wing panels at a low level in the case of the DIA. I. e., the controllers with uncoupled gains showed a better compromise between performance and control energy than the coupled ones. 

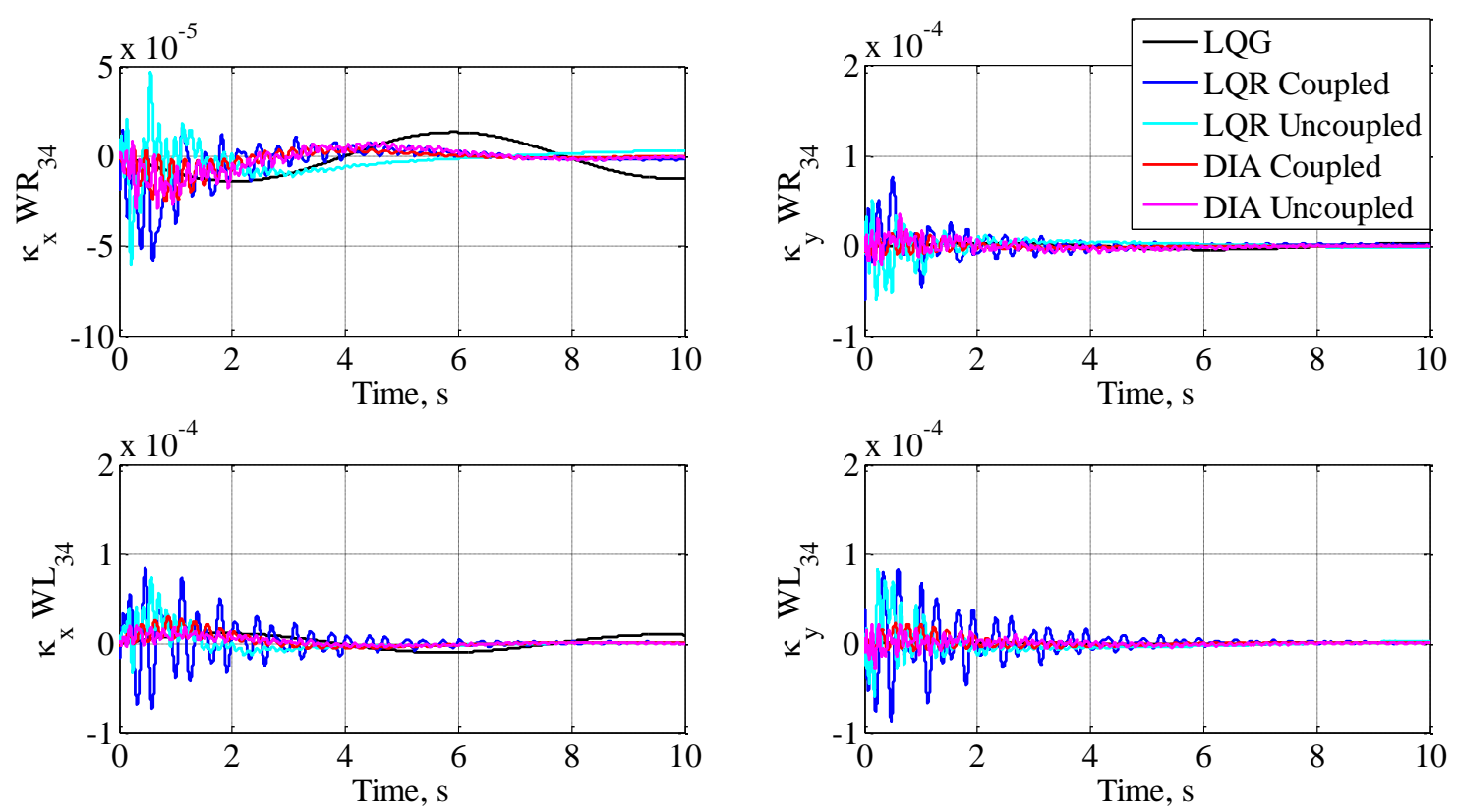

Figure 9. Strains in the central panels of the wing.
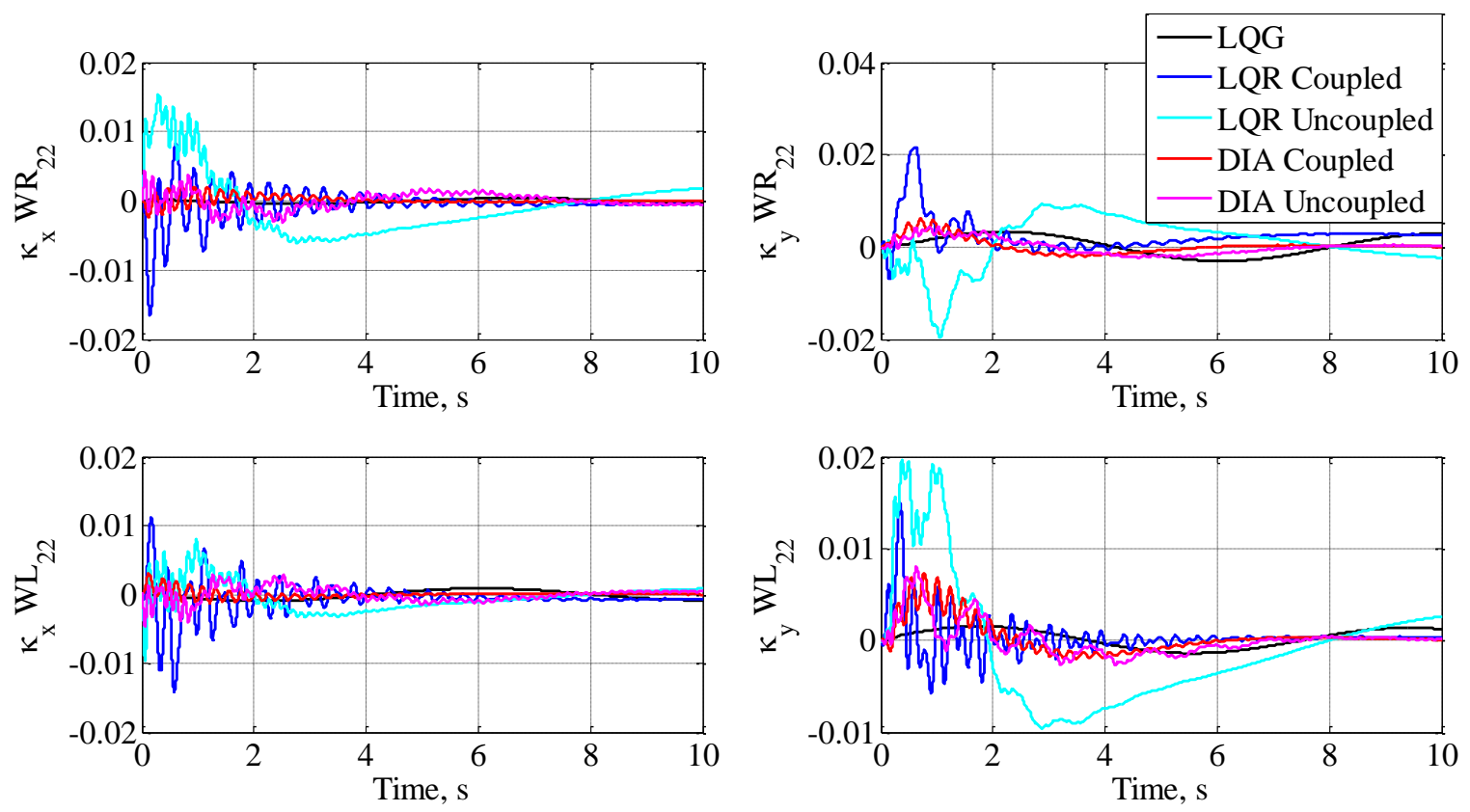

Figure 10. Strains in the middle panels of the wing. 

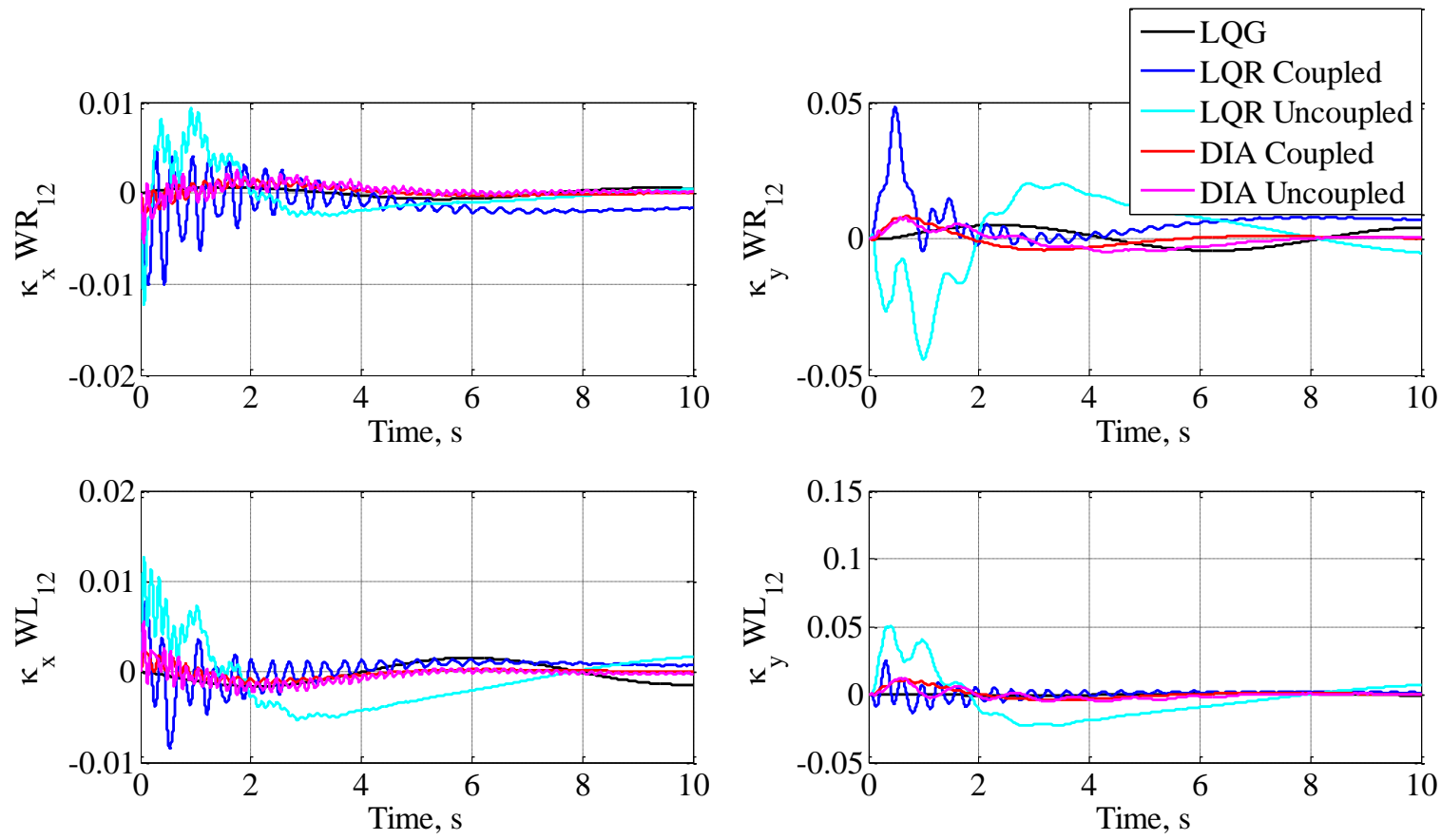

Figure 11. Strains in the outer panels of the wing.

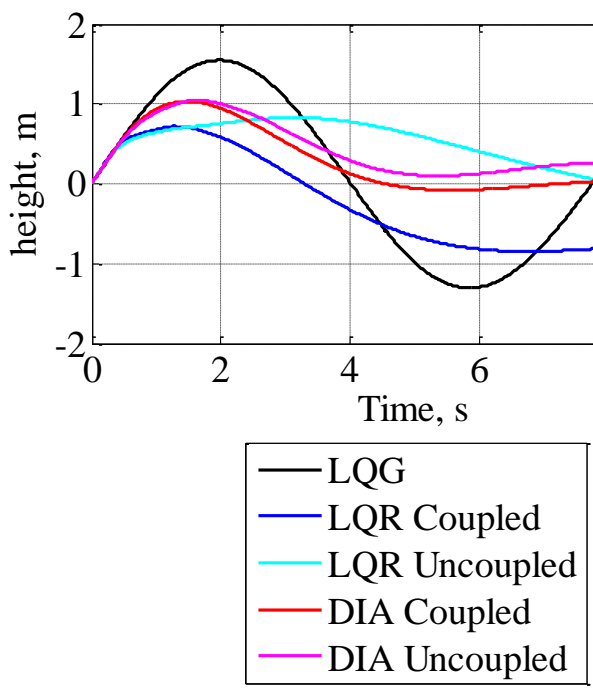

Figure 12. Variation of height in time domain.

12

American Institute of Aeronautics and Astronautics 


\section{Outer-loop}

The outer-loop for the X-HALE has the objective of tracking commanded speed, heading, altitude, and zerosideslip angle. The plant/feedback structure applied in classical multivariable approach for aircraft control design usually offers good robustness properties ${ }^{13}$. The tracker solution allows controller dynamics of any structure and defines the control gains that minimize a performance index over the enclosed structure. The tracking commands act over the system presented in Eq. (1). The performance output is:

$$
z=H x
$$

The output must follow a reference $r(t)$. Compensator dynamics is given in state space form by

$$
\begin{aligned}
& \dot{w}=F w+G e \\
& u_{v}=D w+J e
\end{aligned}
$$

where $w(t)$ is the compensator state and $u_{v}(t)$ is the respective command of the outer loop. The tracking error is

$$
e(t)=r(t)-z(t)
$$

The matrices $F, G, D$ and $J$ are selected to include the desired structure in the compensator. The form of the plant control input is

$$
u=-K y-u_{v}
$$

the respective command of the outer loop is $u_{v}(t)$, that result in the satisfactory tracking of $r(t)$. The augmented system is written as

$$
\begin{gathered}
\frac{d}{d t}\left[\begin{array}{l}
x \\
w
\end{array}\right]=\left[\begin{array}{cc}
A & 0 \\
-G H & F
\end{array}\right]\left[\begin{array}{l}
x \\
w
\end{array}\right]+\left[\begin{array}{l}
B \\
0
\end{array}\right] u+\left[\begin{array}{l}
0 \\
G
\end{array}\right] r \\
{\left[\begin{array}{c}
y \\
u_{v}
\end{array}\right]=\left[\begin{array}{cc}
C & 0 \\
-J H & D
\end{array}\right]\left[\begin{array}{l}
x \\
w
\end{array}\right]+\left[\begin{array}{l}
0 \\
J
\end{array}\right] r} \\
z=\left[\begin{array}{ll}
H & 0
\end{array}\right]\left[\begin{array}{l}
x \\
w
\end{array}\right]
\end{gathered}
$$

The control signal is

$$
u=-\left[\begin{array}{ll}
K & I
\end{array}\right]\left[\begin{array}{c}
y \\
u_{v}
\end{array}\right]
$$

Equations from Eq. (24) to Eq. (26) are able to include the dynamic of the compensator in Eq. (1).

$$
\begin{aligned}
& \dot{x}_{a}=A_{a} x_{a}+B_{a} u_{a}+G r \\
& y_{a}=C_{a} x_{a}+F r
\end{aligned}
$$

The objective of the optimization process is to calculate the gain $K$ to attain the reference. The computation of $K$ is done by closing all feedback loops simultaneously one over the other. The close loop system is 


$$
\dot{x}_{a}=(A-B K C) x_{a}+(G-B K F) r
$$

Different tries were done to obtain the feedback gains using stabilization methods based on Lyapunov or Riccati equations, resulting in poor performance. This confirms the statements by Bompart $e a^{a l^{16,17}}$, Apkarian $e a^{18}$ and Denieul et $a l^{19}$ that LQR methods are insufficient for large and complex systems. We use instead a nonsmooth optimization technique in the frequency domain, which we describe in the following.

\section{A. Nonsmooth optimization technique}

Fixed structure or $H_{\infty}$ tuning is a technique that uses nonsmooth optimizers with design requirements as $H_{\infty}$ constraints in order to provide a solution usually comparable to a full order $H_{\infty}$ synthesis solution. One of the advantage of this technique is the simplicity and the reduced order of the resulting control structure. ${ }^{18}$

Normally, the design specifications for $H_{\infty}$ control synthesis are in the frequency domain and consist in adding restrictions over the sensitivity function $S_{(s)}$, cosensitivity function $T_{(s)}$ and the open loop $P G_{(s)} K_{\text {ext(s) }}$ were $P G_{(s)}$ is the plant to be controlled and $K_{\text {ext(s) }}$ is the feedback/feedforward compensator ${ }^{15}$. The $H_{\infty}$ constrains can be written for any function of the feedback loop, including all sensitivity functions mentioned. The functions of sensitivity and cosensitivity are defined as

$$
\begin{gathered}
S_{(s)}^{\stackrel{\text { def }}{=}}\left(I+P G K_{e x t}\right)^{-1} \\
T_{(s)} \stackrel{\text { def }}{=} P G \cdot K_{e x t}\left(I+P G \cdot K_{e x t}\right)^{-1}=\left(I+P G \cdot K_{e x t}\right)^{-1} P G \cdot K_{e x t}
\end{gathered}
$$

The standard $H_{\infty}$ synthesis problem is presented in Fig. 13. In this figure, the block diagram is the same of a traditional $H_{\infty}$ problem, the difference consisting in the fixed structure controller.

The state vector is $x_{p}$, the exogenous input vector is $e, u$ is the control input vector and $K_{e x t}$ the feedback controller. Regulated output vector is $z$ and the measured output is $y$. Equation 32 shows typical representation of $P G(s)$

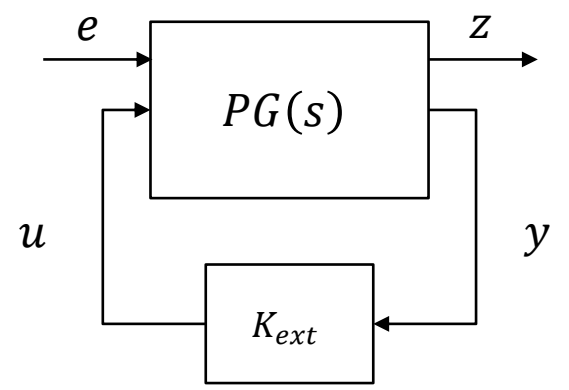

Figure 13. Closed-loop interconnection

$$
P G:\left\{\begin{array}{c}
\dot{x}_{a}=A x_{a}+B_{a} u_{a}+G r \\
z=H x_{a} \\
y_{a}=C x_{a}+F r
\end{array}\right.
$$

The feedback controller is of the form

$$
K_{e x t}:\left\{\begin{array}{l}
\dot{w}=F w+G e \\
u_{v}=D w+J e
\end{array}\right.
$$


It is called structured if the real matrices $F, G, D$ and $J$ depend smoothly on a design parameter $x \in \mathbb{R}^{n}$. Which is a term of tunable parameter ${ }^{20}$. Shorting the notation $K_{\text {ext }(x)}$ to accentuate that the controller is structured with $x$ as tunable element. The differentiable mapping is:

$$
F_{K}=F_{K}(x), G_{K}=G_{K}(x), D_{K}=D_{K}(x), J_{K}=J_{K}(x)
$$

The optimization of the structured controller problem is

$$
\begin{array}{cc}
\text { minimize } & \left\|T_{e z}\left(P G, K_{\text {ext }}\left(x_{\text {tun }}\right)\right)\right\| \\
\text { subject to } & K_{\text {ext }}(x) \text { closedloopstabilizing } \\
& K_{\text {ext }}(x) \text { structured }
\end{array}
$$

Where $T_{e z}\left(P G, K_{e x t}\right)=\widetilde{F}_{l}\left(P G, K_{e x t}\right)$ is the lower feedback connection between Eq. (33) and Eq. (32), which is also called linear fractional transformation ${ }^{20}$. The norm $\|\cdot\|$ in Eq. (35) refers to any $H_{\infty}$ or $H_{2}$ norm. Meanwhile the variable $x \in \mathbb{R}^{n}$ regroups the tunable parameters in the design during the optimization process. ${ }^{20}$

The optimization of the gain for the outer-loop of the X-HALE was done with Systune, a modern control tool available in Matlab Robust Control System Toolbox ${ }^{18,20}$.

The objective of the optimization algorithm is to tune the parameters of Eq. (33) to enforce the closed loop stability. Systune solves multi objective constrained optimization problem with soft and hard constraints of the form

$$
\begin{array}{ll}
\text { minimize } & f\left(x_{\text {tun }}\right)=\max _{k \in S O F T, i \in I_{K}}\left\|T_{\text {eizi }}^{(k)}\left(K_{\text {ext }}\left(x_{\text {tun }}\right)\right)\right\| \\
\text { subject to } & g\left(x_{\text {tun }}\right)=\max _{k \in H A R D, j \in I_{K}}\left\|T_{\text {ejzj }}^{(k)}\left(K_{\text {ext }}\left(x_{\text {tun }}\right)\right)\right\| \leq 1 \\
& K_{\text {ext }}\left(x_{\text {tun }}\right) \text { structured and stabilizing }
\end{array}
$$

Where $T_{e 1 z i}^{(k)}$ is the $i$ th close loop robustness or performance channel $e_{i \rightarrow z_{i}}$ for $k$ th plant model $P G$. The goal of Eq. (36) is to minimize the worst case cost of the soft constraints while enforcing the hard constraints and close loop stability. ${ }^{20}$

The designs requirements of the controller are based on the ability of the aircraft to attain the commanded reference, while all the requirements of the inner loop are kept. An iterative process was developed to construct a proper controller. Constrains were applied on $P G \cdot K$ functions, the open loop cut off frequencies were set between 0.25 and $1 \mathrm{rad} / \mathrm{sec}$. The idea was to reach to the physical limit of the aircraft actuators, the constraints were tight until the optimization process was capable to reach the desired performance.

A four-controller configuration was proposed for the X-HALE. Two central elevators give control input for the velocity controller. The bank angle is controlled with the two outer elevators acting together differentially. The sideslip angle is regulated with the differential thrust of the outer engines acting a pair minus the other and the height is commanded with the five engines. The control variables for the outer loop are presented in Eq. (37).

$$
u_{v}=\left[\begin{array}{llll}
\eta_{T R 2}+\eta_{T L 1} & \eta_{T R 4}-\eta_{T L 2} & \eta_{F 2}+\eta_{F 3}-\left(\eta_{F 1}+\eta_{F 4}\right) & \eta_{F 0}+\eta_{F 1}+\eta_{F 2}+\eta_{F 3}+\eta_{F 4}
\end{array}\right]^{T}
$$

The feedback controller $K_{(x)}$ is chosen as a 10th-order state-space system, thereby ensuring controller simplicity as required by specification. There are eight compensators for speed, height, sideslip and bank angle and two compensators for heading.

\section{Outer-loop Results}

Three maneuvers were commanded on the controller. Maneuver 1 consists in a wing-level altitude change of 5 $\mathrm{m}$. Maneuver 2 is a steady levelled turn with zero sideslip and 90 degrees heading change. Maneuver 3 is a climbing turn that combines the features of the other two maneuvers. The results shown in the following are based on XHALE linearized dynamics. 


\section{A. Maneuver 1}

Figures 14 to 17 present simulation results for the first simulation case. In Fig. 14, it is observable how the airplane is capable to track the new height with a maximum overshoot of 9.83 percent. The stationary error state is 1.4 percent. While the controller is trying to attain the commanded height the aircraft presents a heading variation, inducing a roll angle change. When the maneuver is completed the control system is capable to reduce this deviation. Once the aircraft has achieved the commanded altitude all lateral perturbations are damped. It is possible to note how the sideslip angle is controlled during the entire maneuver with a maximum variation of 0.2 degrees. The aircraft velocity start is reduced during the beginning of the maneuver, it reach a lowest value $12.75 \mathrm{~m} / \mathrm{s}$. After 6 seconds, it starts to return to the trim velocity of $14 \mathrm{~m} / \mathrm{s}$. The final stationary error state is 0.35 percent.

In Fig. 15 the angular rates are presented. It is appreciable the small magnitude of this variation rates during the entire maneuver and how they are smoothly damped by the controller. The maximum variation of the pitch angle during the maneuver is of 7.2 degrees and the maximum angle of attack is 2.2 degrees.

The control action is plotted on Fig. 16. The controller are not saturated in any moment during the maneuver, the inner right elevator presents the maximum variation of the control surfaces with 10.71 degrees. It is interesting to point out that the central elevators are activated symmetrically in the initial part of the maneuver; also, the outer elevators are deflecting symmetrically to sustain the commanded heading. Meanwhile the engines are acting to attain the commanded speed symmetrically which could confirm that the action of the controller does not have any responsibility on the heading variation. Figure 17 shows the variation of strains during the maneuver. This figure present the controlled bending strains distributed over the structure for both wings. They are plotted one over the
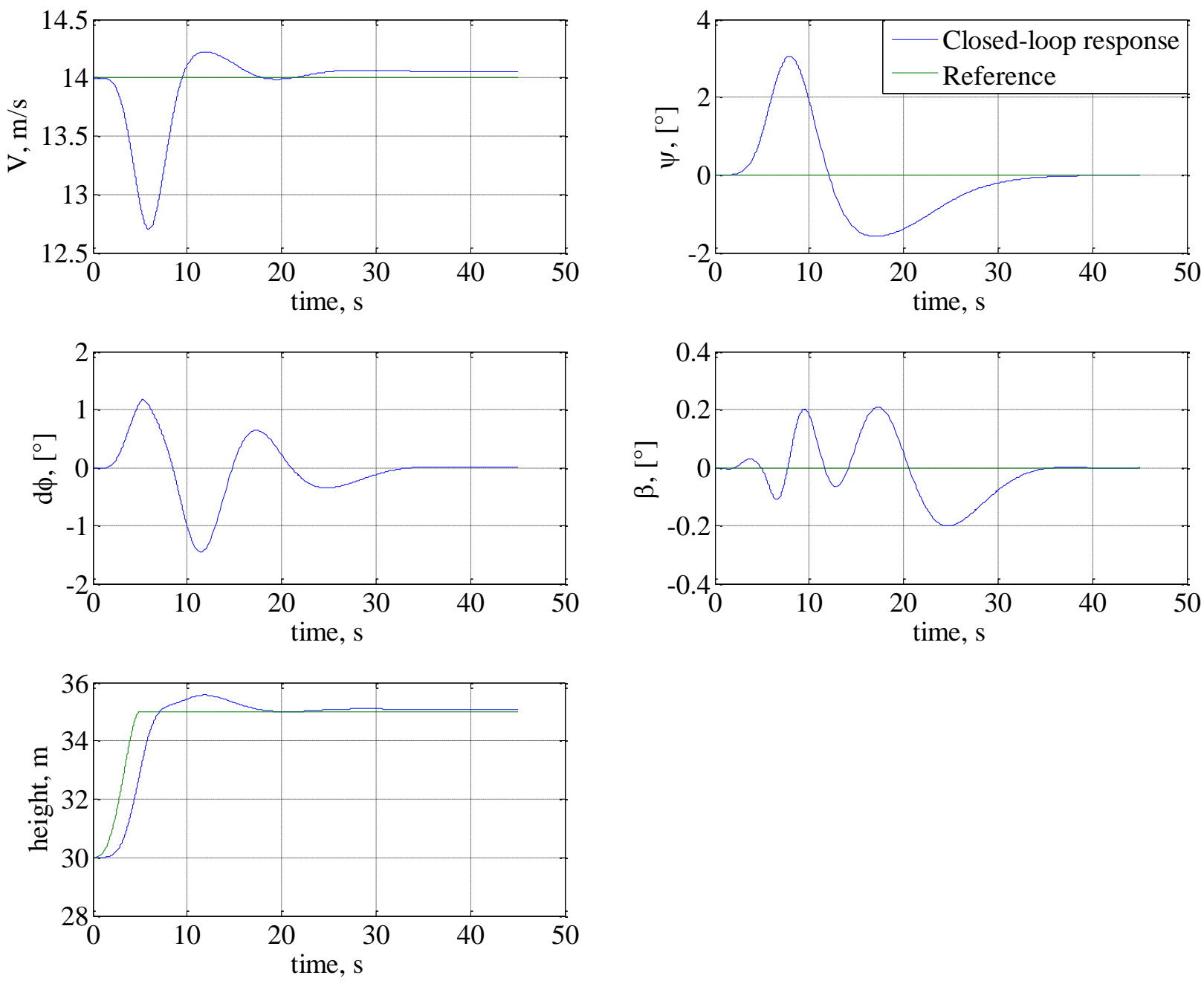

Figure 14. Tracked reference for first outer loop simulation 
other for comparison. It is possible to appreciate that since the beginning of the maneuver, the structure is asymmetrically deformed, after the first 10 seconds the dynamics of the structure is controlled and reduced to the equilibrium condition. Both external elevators are acting to hold the deformation level and to reduce the heading variation. After the commanded altitude is attained, it is possible to note how the engines are used to reduce the sideslip angle and then return to the initial condition. It is possible to affirm that this lateral motion variation was induced by the aircraft asymmetry. However is interesting to point how the structure is not significantly deformed during this maneuver, Fig. 17 illustrates the deformation of the structure during the maneuver.
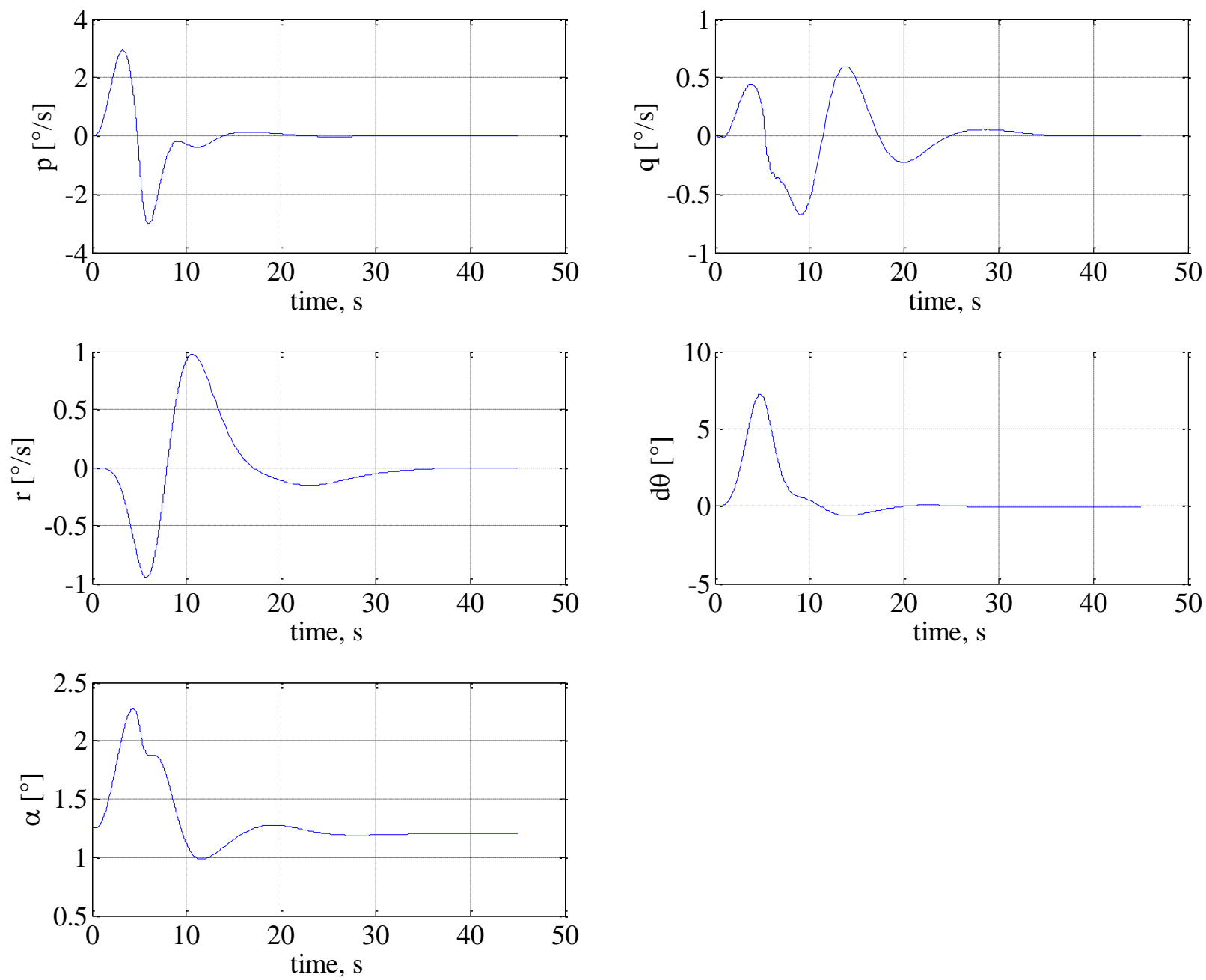

Figure 15. Angular rates, pitch angle and angle of attack for first outer loop simulation 


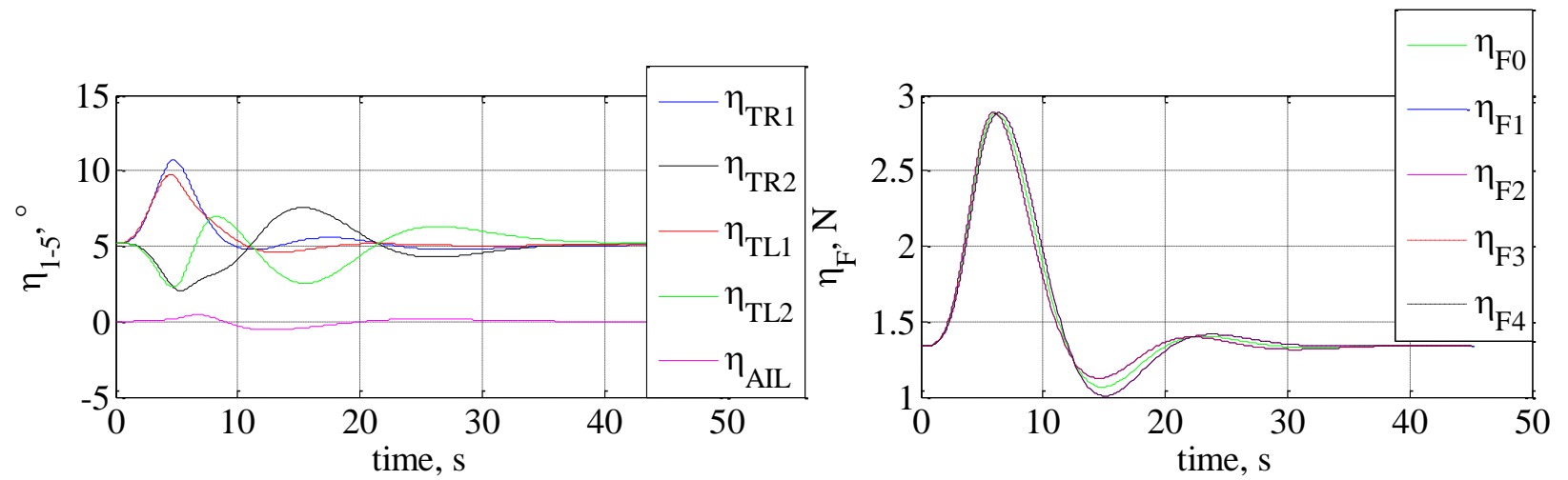

Figure 16. Variation of control inputs for first outer loop simulation

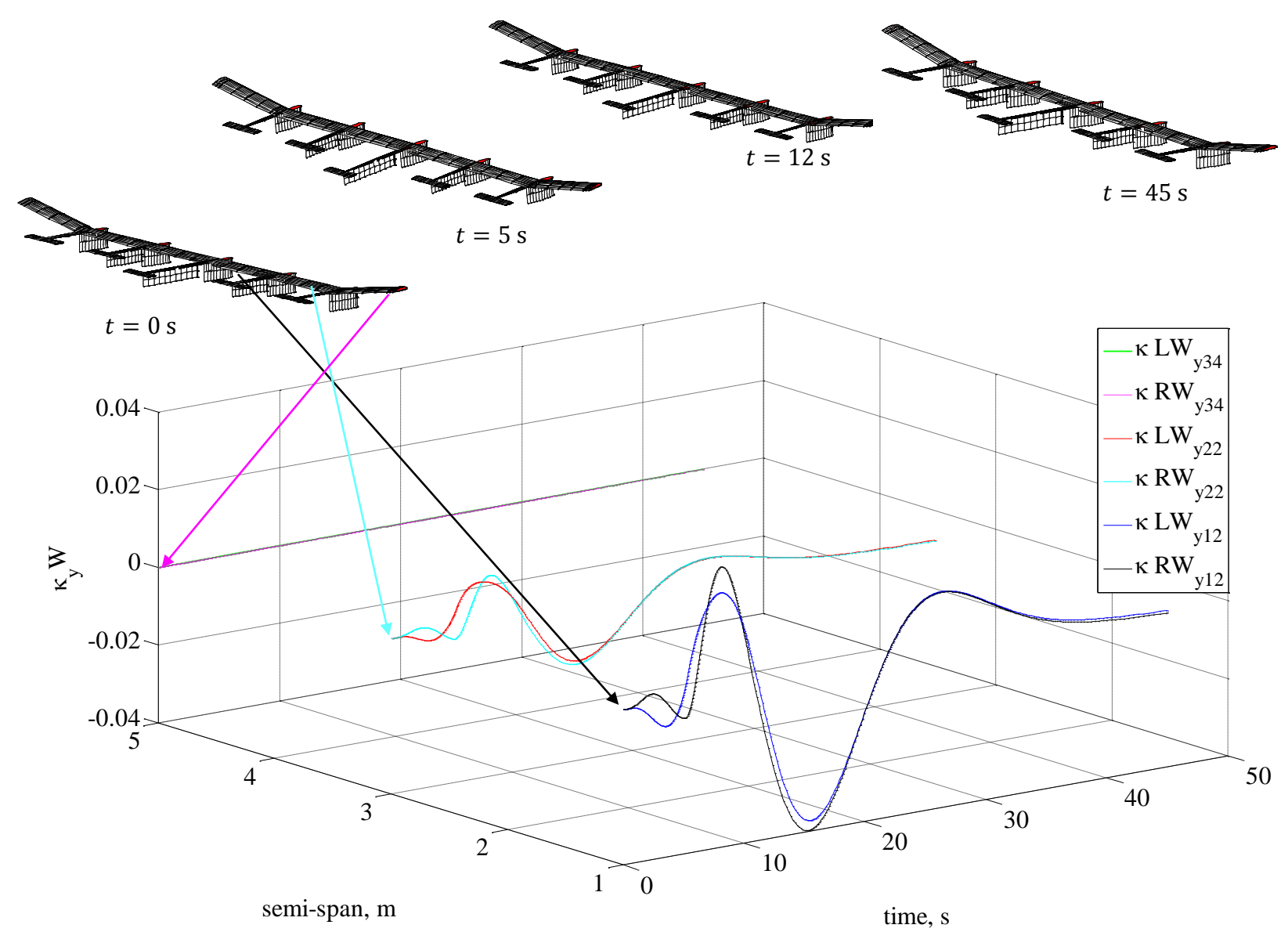

Figure 17. Variation of the bending wing strains for first outer loop simulation 


\section{B. Maneuver 2}

The second maneuver consist in a coordinated turn of ninety degrees at constant altitude and speed. The tracked states are presented in Fig. 18. The X-HALE starts at its trim condition and after the reference is commanded it start to turns. The heading tracker does not present any overshoot and the final state error is -0.49 percent. The heading command generates a roll angle command that makes the aircraft turn. The maximum roll angle reached was 11.23 degrees. Once it starts to attain the reference, the aircraft returned to the level wing condition. It practically did not change speed nor altitude. In addition, the sideslip angle is hold between 0.54 and -0.35 degrees to ensure the coordinate turn.

Additionally, it is interesting to point out how the angle of attack presented in Fig. 19 is increased to hold the coordinated turn. The pitch angle does not change during the maneuver. Equally, the angular rates presented in the same figure have a smooth behavior. $p$ almost does not chance. $q$ and $r$ show how the angular rates for yaw and roll vary during the maneuver and once the command is reached they return to the equilibrium condition.

The controls for lateral command are more demanded in this maneuver. The external left elevator almost reach the saturation limit of 30 degrees, maximum deflection of this control surface was 28.9 degrees. The external elevator are deflected asymmetrically to attend the reference while the rest of the control surfaces act to hold the shape of the aircraft. Internal elevators are deflecting asymmetrically to hold the deformation of the structure with the ailerons. At the same time engines are activated asymmetrically to sustain the side-slip angle. The central engine varies to hold the altitude.
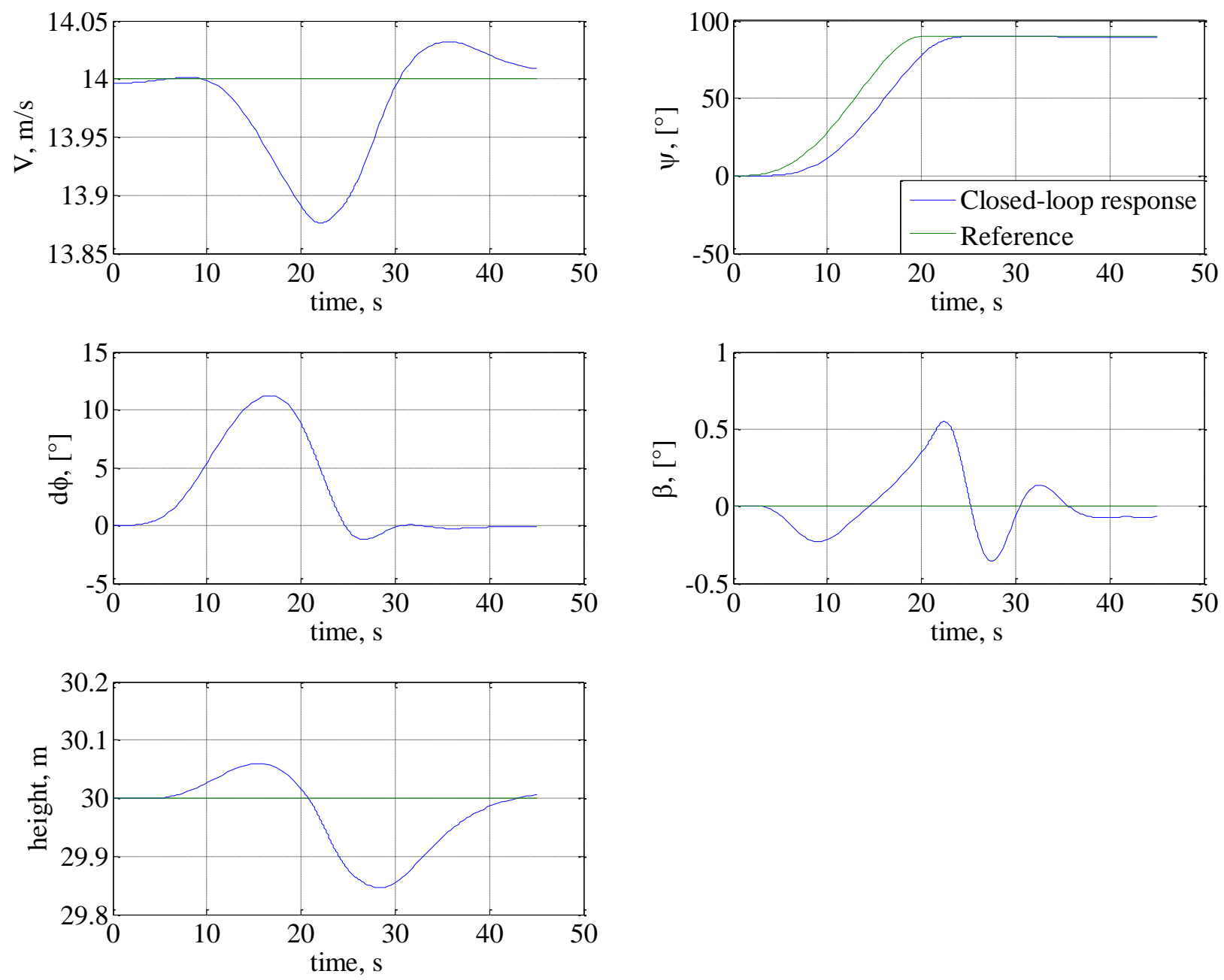

Figure 18. Tracked reference for first outer loop simulation 
It is interesting to comment about the dynamics of the controller in this maneuver. Engines $\mathrm{F}_{2}$ and and $\mathrm{F}_{4}$ are variating in the same rate that $F_{1}$ and $F_{3}$, however once they have reached the commanded heading they remain in a thrust condition different from the initial. The aircraft is capable to hold speed and altitude with a different control action. The total thrust of the engines is the same of the trim condition but it is not equally distributed. In order to hold the variation of strain with this new thrust condition the elevators are also holding the shape of the structure in a position different from trim.

Nevertheless, the strains on the structure are returned to the trim condition and the shape is hold. Figure 21 show the strain increasing in both wings during the maneuver. Once again, there is a little asymmetry between wing strains. The right wing is deforming more that left. The deformed shape of the structure during the maneuver is presented on Fig 21.
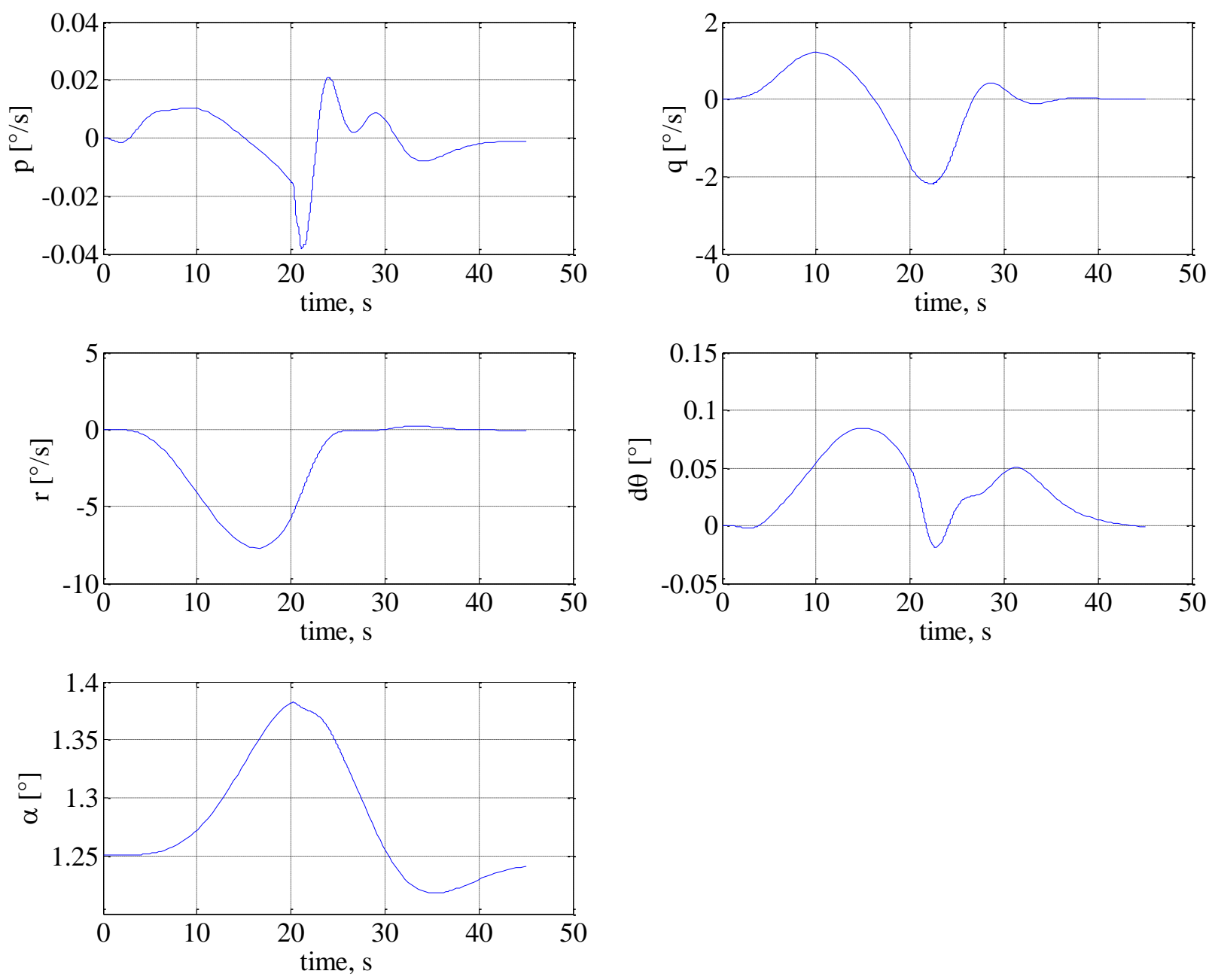

Figure 19. Angular rates, pitch angle and angle of attack for second outer loop simulation 


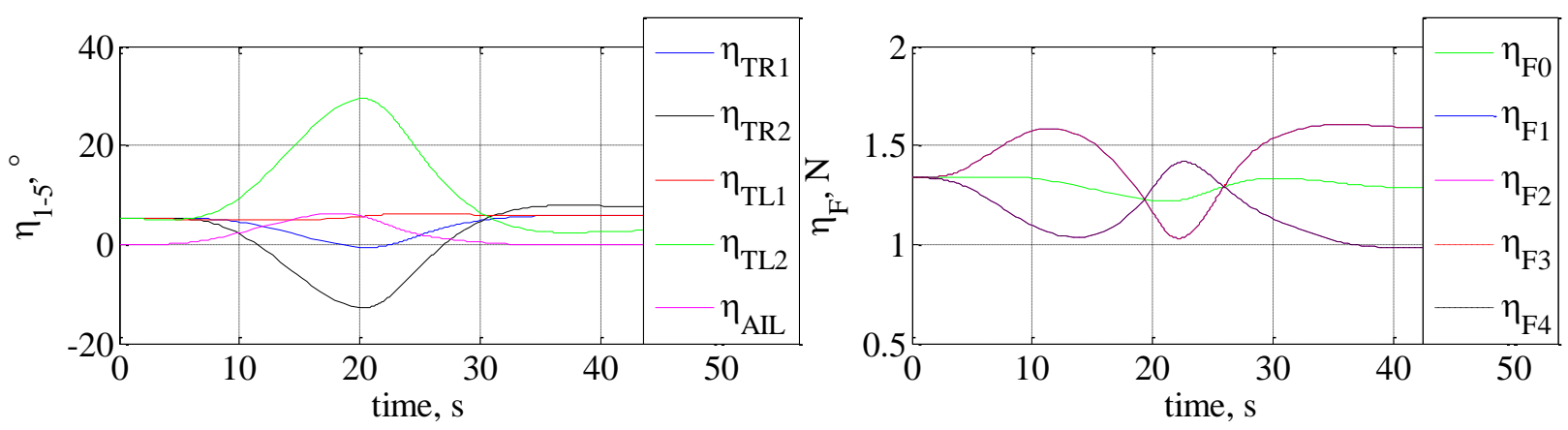

Figure 20. Variation of control inputs for second outer loop simulation

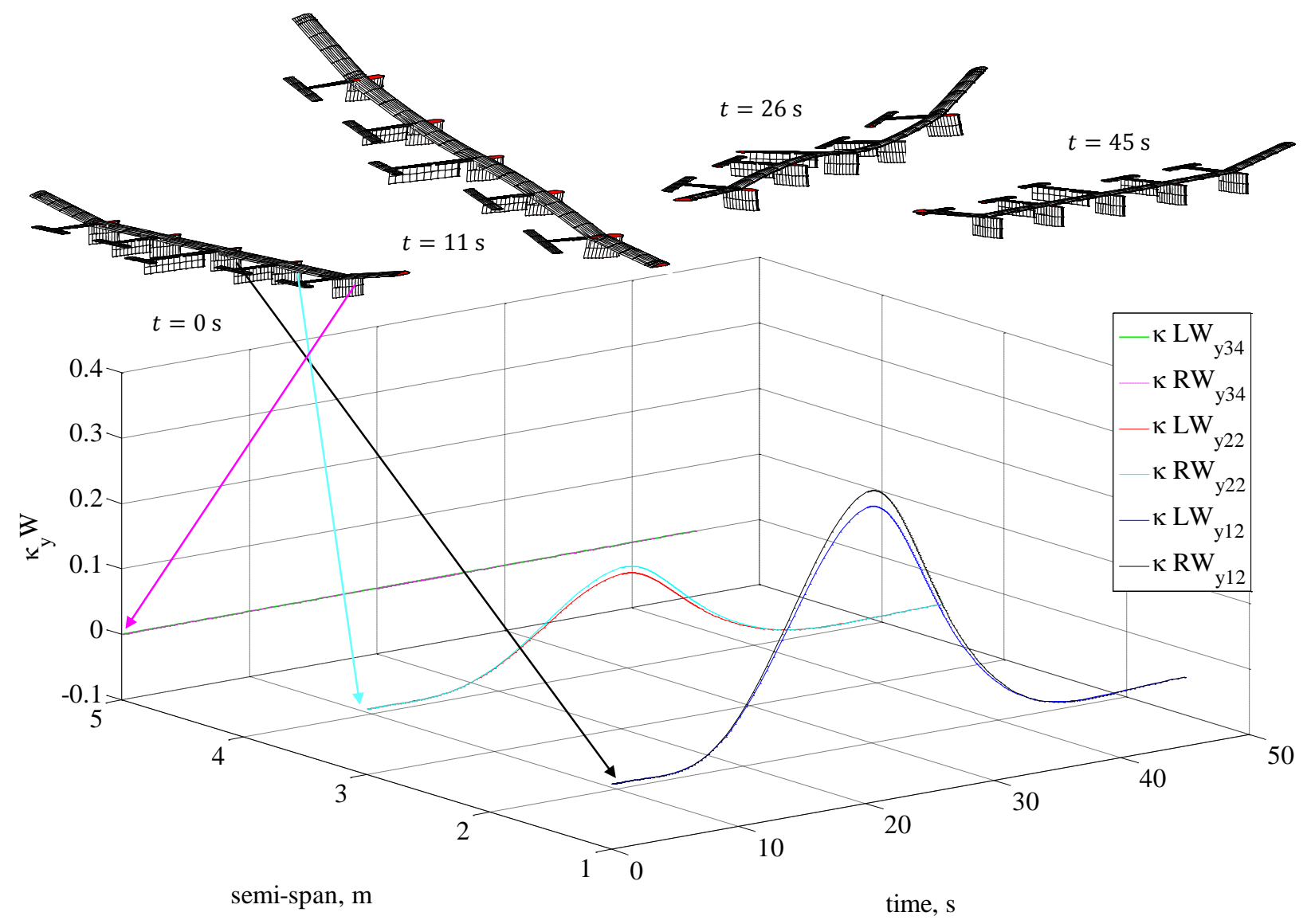

Figure 21. Variation of the bending wing strains for second outer loop simulation 


\section{Maneuver 3}

Last maneuver consists in a variation of height of five meter while a ninety degree coordinated turn is completed. The outer loop is capable to take the X-HALE to both commanded references satisfactorily. The increase of five meter in height is attained with a maximum overshoot of 5 percent. Stationary steady state error of 1.5 percent. The heading reference is reached after 23 seconds and the stationary steady state error is -0.55 percent. The other two references are tracked properly, the aircraft speed does not change more than $0.44 \mathrm{~m} / \mathrm{s}$ and the sideslip angle is hold between 0.61 and -0.33 degrees.

Pitch angle and angle of attack vary to increase the altitude and hold the coordinated turn respectively. The dynamic of the angular rates are presented in Fig. 23. It is possible to observe how the pitch rate is changing to attain the longitudinal requirements for the variation of height. In the meantime, the yaw and roll rates present the dynamic of the aircraft to complete the turn and return to level flight condition. Once both reference are reached, the aircraft return to steady level flight.

Control variation for this maneuver are shown in Fig. 24. The engines increase thrust to attain the commanded altitude, at the same time they generate an asymmetric thrust to hold the sideslip angle as close to zero as possible. Meantime the control surfaces are keeping the shape of the wing and commanding the heading tracking. Once the reference is reached the control surfaces and engines hold the level straight flight condition with a control deflection and engine thrust different from the initial trim condition.

During this maneuver the predominant effect on the deformation of the wing is the turn. The order of strain values is ten times higher compared to Fig. 17. The lateral maneuver has a greater effect in strain increase that the
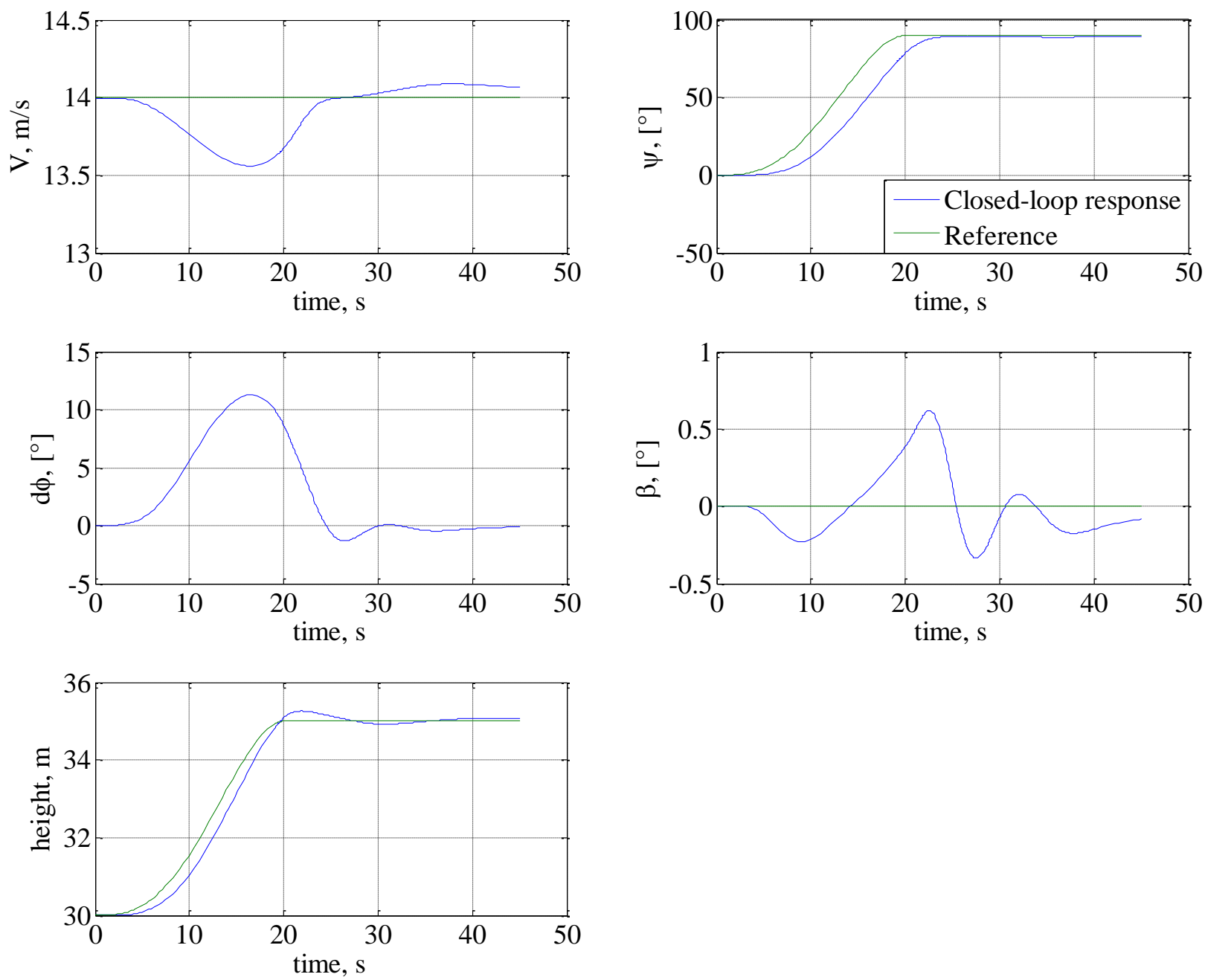

Figure 22. Tracked reference for first outer loop simulation 
longitudinal maneuver. The control system is capable of bringing the structure to its initial strain distribution condition as is seen in the structure illustration of Fig 25.
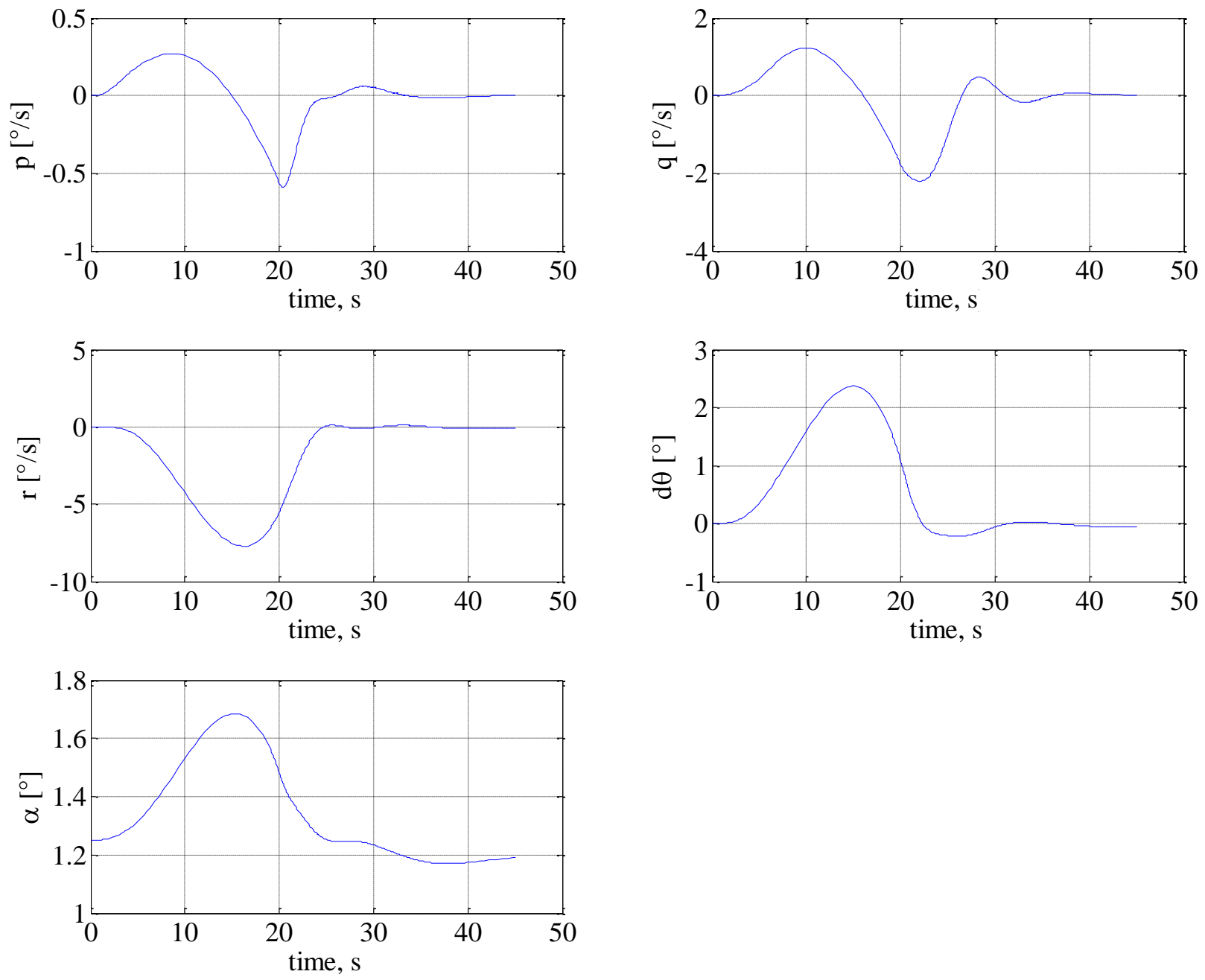

Figure 23. Angular rates, pitch angle and angle of attack for third outer loop simulation 


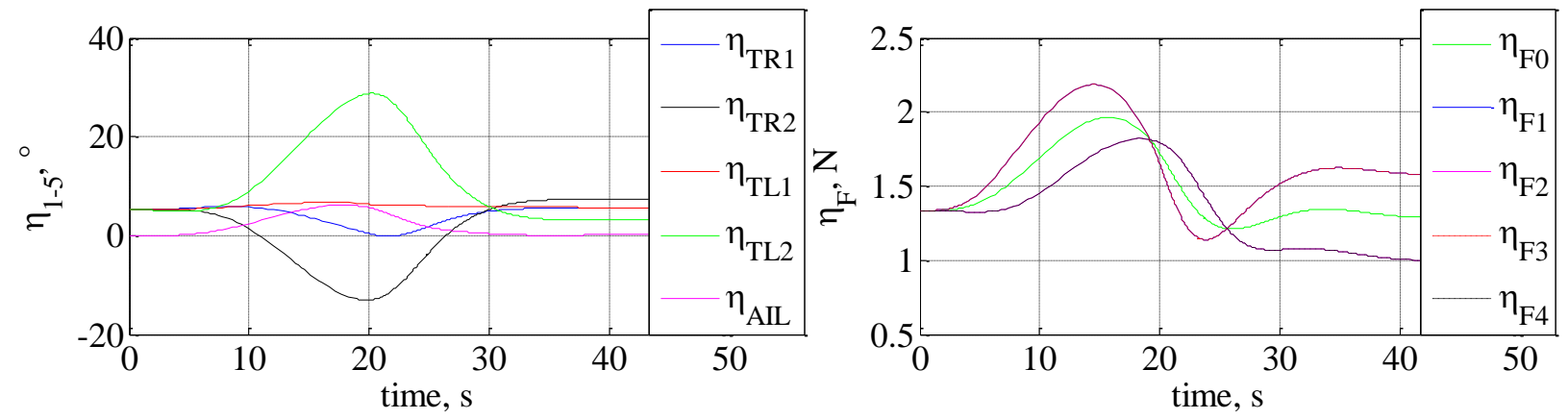

Figure 24. Variation of control inputs for third outer loop simulation

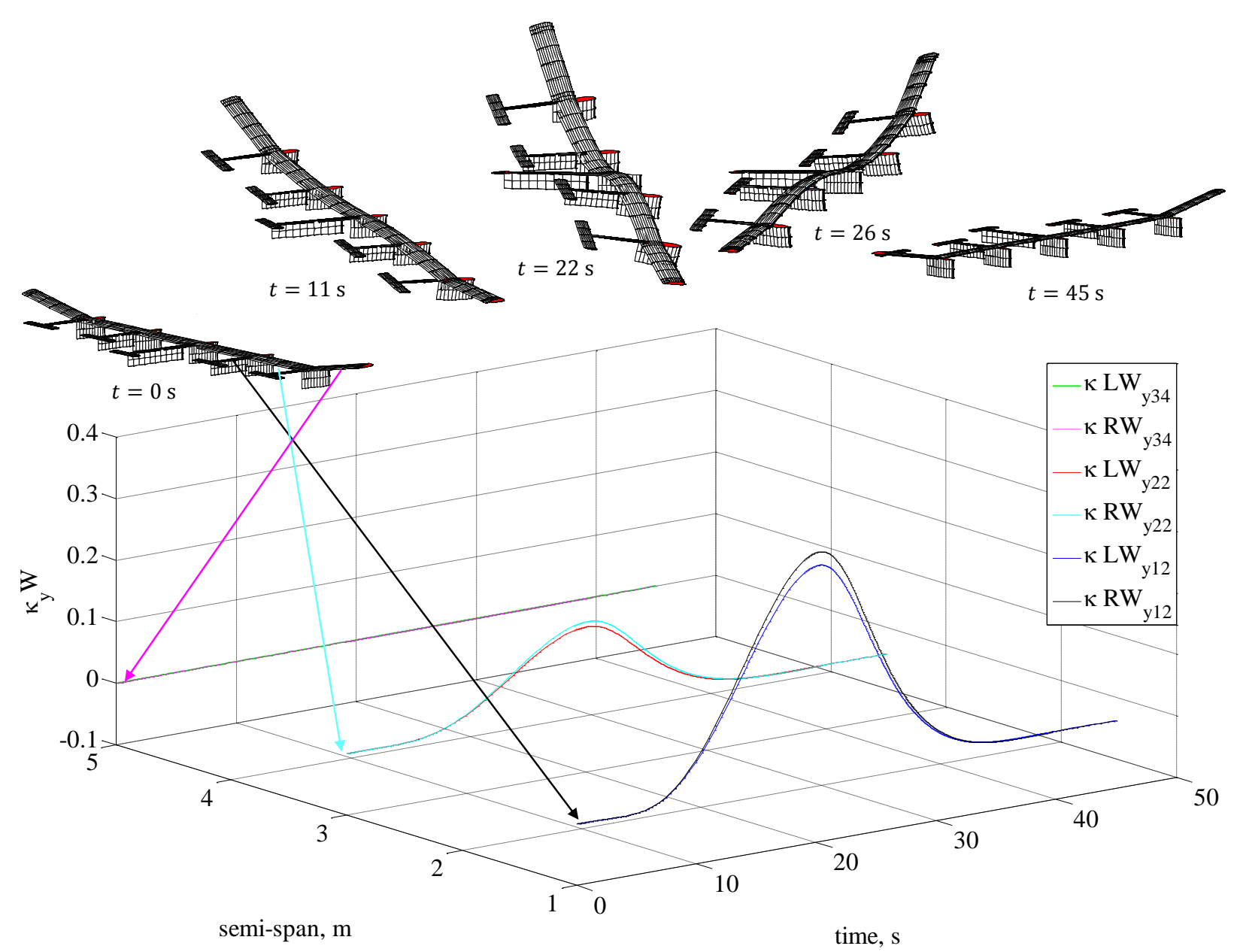

Figure 25. Variation of the bending wing strains for third outer loop simulation

American Institute of Aeronautics and Astronautics 


\section{Conclusion}

A control law for a highly flexible aircraft was proposed and demonstrated for the flexible aircraft X-HALE. The loop separation concept consists in an inner loop capable of holding the shape of the aircraft while ensuring flight stability. The outer loop in this concept is used to track references. In this case, two outer loops are operating to command heading, speed, sideslip angle and altitude. A third outer loop could be implemented to receive waypoint coordinates.

The gains in the inner loop were estimated by three methods. It is possible to observe how the controllers with uncoupled gains have a better response in the time domain than the coupled ones. The uncoupled controllers are capable of bringing X-HALE to the equilibrium point faster, in general with less overshoot and higher damping. Decoupling the gains reduce the control energy to hold equilibrium condition and wing shape.

The outer loop controller consists in a fixed-structure controller. The gains and actuators' bandwidth have been optimized in a single process, and the results demonstrated adequate closed-loop performance while attaining control requirements in terms of maneuverability. The commanded references could be tracked in all simulated cases with low steady state errors and no control saturation. The loop separation concept allows to hold the wing shape in all simulation cases, showing promising results.

\section{Acknowledgments}

The authors would like to thank the financial support from the Brazilian funding agency Financiadora de Estudos e Projetos FINEP and EMBRAER.

\section{References}

${ }^{1}$ Gradient, R., Lavretsky, E. and Wise, K., "Very Flexible Aircraft Control Challenge Problem" AIAA Guidance, Navigation, and Control Conference. AIAA 2012-4973, Minneapolis, Minnesota, United State, 2012.

${ }^{2}$ Tuzcu, I., Marzocca, P., Cestino, E., Romeo, G. and Frulla, G., Stability and Control of a High-Altitude, Long-Endurance UAV. Journal of Guidance, Control, and Dynamics, Vol. 30, No. 3, May-June 2007, 713-721.

${ }^{3}$ Davey, P., "Zephyr HALE UAS", AIAA Infotech Aerospace 2007 Conference and Exhibit, AIAA 2007-28467, Rohnert Park, California, United States, 2007.

${ }^{4}$ Johnstone, R. and Arntz, N., "CONDOR-High Altitude Long Endurance (HALE) Automatically Piloted Vehicle". AIAA/AHS/ASEE Aircraft Design, Systems and Operations Conference. AIAA-90-3279-CP, Dayton, Ohio, United State, 1990.

${ }^{5}$ Cesnik, C., Palacios, R. and Reichenbach E., "Reexamined Structural Design Procedures for Very Flexible Aircraft” Journal of Aircraft. Vol. 51, No. 5, Sept-Oct, 2014, pp 1580-1591.

${ }^{6}$ Noll,T.E.,Brown,J.M.,Perez-Davis,M.E.,Ishmael,S.D.,Tiffany,G. C., and Gaier, M., "Investigation of the Helios Prototype Aircraft Mishap. Vol. 1: Mishap Report" [online article], http://www.nasa.gov/pdf/64317main_helios.pdf [retrieved 15 Oct. 2015].

${ }^{7}$ S. Cesnik, C., Senatore, P., Su, W., Atkins, E., Shearer, C., X-HALE: A Very Flexible Unmanned Aerial Vehicle for Nonlinear Aeroelastic Tests. AIAA Journal Vol. 50, No. 12, Dec 2012, pp 2820-2833.

${ }^{8}$ Jones, J. and Cesnil, C., "Preliminary Flight Test Correlations of the X-HALE Aeroelastic Experiment". International Forum on Aeroelasticity \& Structural Dynamics (IFASD-2013), Bristol, United Kingdom, 2013.

${ }^{9}$ Pang, Z., Cesnik, C. and Atkins, E., "In-Flight Wing Deformation Measurement System for Small Unmanned Aerial Vehicles" 55th AIAA/ASMe/ASCE/AHS/SC Structures, Structural Dynamics, and Materials Conference AIAA SciTech 2014, AIAA 2014-0330, National Harbor, Maryland, United States, 2014.

${ }^{10}$ Dillsaver, M. "Gust Response and Control of Very Flexible Aircraft" PhD Thesis, Aerospace Engineering Department, University of Michigan. Ann Arbor, Michigan, United States, 2013.

${ }^{11}$ Specification, M., "Flying qualities of piloted aircraft" Technical report, MIL-1797A, 19 of December 1997.

${ }^{12}$ Shearer, C. and Cesnik, C. "Trajectory control for very flexible aircraft", Journal of Guidance Control and Dynamics, Vol. 31, No 2, 2008, pp 340-357.

${ }^{13}$ Dillsaver, M., Cesnik, C. and Kolmanovsky, I., "Trajectory Control of very flexible Aircraft with Gust Disturbance", Guidance, Navigation, and Control and Co-located Conferences/ AIAA Atmospheric Flight Mechanics (AFM) Conference, AIAA 2013-4745 Boston, Massachusetts, United States, 2013.

${ }^{14}$ Stevens, B. and Lewis, F., Aircraft control and simulation, John Wiley \& Sons, 2003.

${ }^{15}$ Skogestad, S. and Postlethwaite, I., Multivariable feedback control analysis and design, Second edition, John Wiley \& Sons, 2005.

${ }^{16}$ Bompart, V., Apkarian, P. and Noll, D., "Non-smooth techniques for stabilizing linear systems", American Control Conference, WeB16.4, New York City, United States, 2007

${ }^{17}$ Bompart, V., Apkarian, P. and Noll, D., "Control design in the time frequency domain using nonsmooth techniques". Systems \& Control Letters, No 57, pp 271-282, 2008.

${ }^{18}$ Apkarian, P., Noll, D., "Nonsmooth $H_{\infty}$ Synthesis”, IEEE Transactions on Automatic Control, Vol. 51, No. 1, 2006. 
${ }^{19}$ Denieul, Y., Bordeneuve, J., Alazard, D., Toussaint, C and Taquin, G., "Integrated Design and Control of a FlyingWing Using Nonsmooth Optimization Techniques", Proceedings of the 3rd CEAS EuroGNC, Specialist Conference on Guidance, Navigation \& Control, Toulouse, France, 2015.

${ }^{20}$ Apkarian P., and Noll, D. "Optimization-Based Control Design Techniques and Tools", Encyclopedia of Systems and Control, Springer London, pp. 1-12, 2014 